\title{
Simultaneous confidence intervals for all pairwise differences between the coefficients of variation of rainfall series in Thailand
}

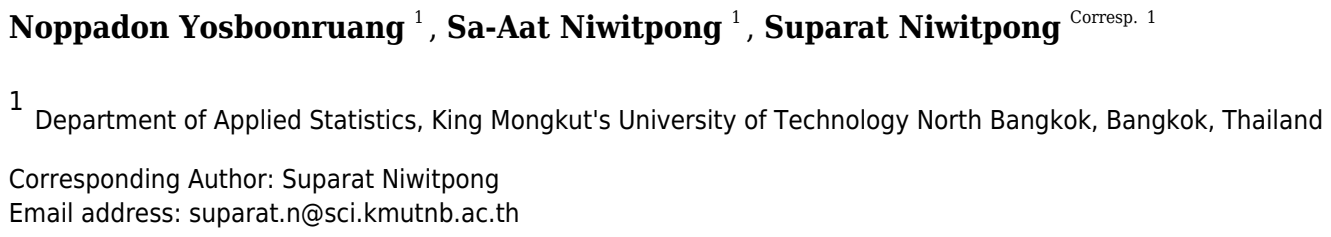

The delta-lognormal distribution is a combination of binomial and lognormal distributions, and so rainfall series that include zero and positive values conform to this distribution. The coefficient of variation is a good tool for measuring the dispersion of rainfall. Statistical estimation can be used not only to illustrate the dispersion of rainfall but also to describe the differences between rainfall dispersions from several areas simultaneously. Therefore, the purpose of this study is to construct simultaneous confidence intervals for all pairwise differences between the coefficients of variation of delta-lognormal distributions using three methods: fiducial generalized confidence interval, Bayesian, and the method of variance estimates recovery. Their performances were gauged by measuring their coverage probabilities together with their expected lengths via Monte Carlo simulation. The results indicate that the Bayesian credible interval using the Jeffreys' rule prior outperformed the others in virtually all cases. Rainfall series from five regions in Thailand were used to demonstrate the efficacies of the proposed methods. 
Simultaneous confidence intervals for all . pairwise differences between the . coefficients of variation of rainfall series in "Thailand

${ }_{5}$ Noppadon Yosboonruang $^{1}$, Sa-Aat Niwitpong ${ }^{1}$, and Suparat Niwitpong ${ }^{1}$

\author{
${ }^{1}$ Department of Applied Statistics, King Mongkut's University of Technology North \\ Bangkok, Bangkok, Thailand \\ Corresponding author: \\ Suparat Niwitpong ${ }^{1}$ \\ Email address: suparat.n@sci.kmutnb.ac.th
}

\begin{abstract}
The delta-lognormal distribution is a combination of binomial and lognormal distributions, and so rainfall series that include zero and positive values conform to this distribution. The coefficient of variation is a good tool for measuring the dispersion of rainfall. Statistical estimation can be used not only to illustrate the dispersion of rainfall but also to describe the differences between rainfall dispersions from several areas simultaneously. Therefore, the purpose of this study is to construct simultaneous confidence intervals for all pairwise differences between the coefficients of variation of delta-lognormal distributions using three methods: fiducial generalized confidence interval, Bayesian, and the method of variance estimates recovery. Their performances were gauged by measuring their coverage probabilities together with their expected lengths via Monte Carlo simulation. The results indicate that the Bayesian credible interval using the Jeffreys' rule prior outperformed the others in virtually all cases. Rainfall series from five regions in Thailand were used to demonstrate the efficacies of the proposed methods.
\end{abstract}

\section{INTRODUCTION}

Thailand is located in Southeast Asia and is classed as a tropical area. It is influenced by both the southwest and northeast monsoons. The southwest monsoon crosses Thailand between mid-May to mid-October (the rainy season) and brings moist air from the Indian Ocean that causes clouds and heavy rain. The northeast monsoon crosses Thailand from mid-October to mid-February (the winter season) causing cold and dry weather. Moreover, the South receives additional heavy rainfall due to moisture coming in from the Gulf of Thailand. The season changes from mid-February to mid-May (the summer season) due to uncertainty in the weather and is influenced by tropical cyclones in the South China Sea, and thus, the weather is generally hot and dry but often with heavy rain and thunderstorms (Thai Meteorological Department, 2015). Thailand often endures flooding due to thunderstorms, which can take lives and damage property, especially on farms due to Thailand being an agricultural country. Thailand is divided into five regions according to its climate pattern and meteorological conditions (Table 1) (Thai Meteorological Department, 2015). Therefore, it is important to investigate rainfall dispersion in each area to gain preliminary information for formulating policies to mitigate such incidents.

There have been numerous studies on rainfall series that have used the delta-lognormal distribution. Fukuchi (1988) derived the distribution of correlation coefficients of rainfall rates from two areas as bivariate lognormal (delta-lognormal). Kedem (1990) showed that the average rain rate over an area follows a delta-lognormal distribution. Shimizu (1993) and Kong et al. (2012) presented the maximum likelihood estimation of the parameters of rainfall series containing zeros that followed a bivariate lognormal distribution. Moreover, examples of rainfall series that conform to a delta-lognormal distribution can be founded in various studies by Maneerat et al. (2019a,b, 2020a,b), Yosboonruang et al. (2019b, 2020), and Yosboonruang and Niwitpong (2020). In addition, a delta-lognormal distribution has been 
Table 1. The provinces of each regions in Thailand.

\begin{tabular}{ll}
\hline \multicolumn{1}{c}{ Regions } & \multicolumn{1}{c}{ Provinces } \\
\hline Northern & Chiang Rai, Mae Hong Son, Chiang Mai, Phayao, Lamphun, Lampang, Phrae, Nan, \\
& Uttaradit, Phitsanulok, Sukhothai, Tak, Phichit, Kamphaeng Phet, Phetchabun
\end{tabular}

applied in other fields, such as Ingram Jr. et al. (2010), Owen and DeRouen (1980), Fletcher (2008), Wu and Hsieh (2014), and Zhou and Tu (2000). Constructing the confidence intervals for several parameters of a delta-lognormal distribution used in statistical inference has been of interest to many researchers. Confidence intervals for the delta-lognormal mean were proposed by Owen and DeRouen (1980), Kvanli et al. (1998), Zhou and Tu (2000), Tian (2005), Chen and Zhou (2006), Tian and Wu (2006), Fletcher (2008), Li et al. (2013), Wu and Hsieh (2014), Hasan and Krishnamoorthy (2018), and Maneerat et al. (2018, 2019a,b). Furthermore, the confidence intervals for variance and the coefficient of variation (CV) of a delta-lognormal distribution were presented by Buntao and Niwitpong (2012, 2013), Yosboonruang et al. (2018, 2019a,b, 2020), Yosboonruang and Niwitpong (2020), and Maneerat et al. (2020a,b).

For statistical inference, the $\mathrm{CV}$, the ratio of the standard deviation to the mean, is a good tool for investigating rainfall dispersion. The advantage of using the $\mathrm{CV}$ is that it is unitless and thus, is useful for measuring dispersion in data series with different units or drastically different means. Focusing on inferential statistics, the confidence intervals and functions of the CV for several distributions have been presented. Wong and $\mathrm{Wu}(2002)$ suggested a small-sample asymptotic method for constructing the confidence intervals for the $\mathrm{CV}$ of normal and non-normal distributions when the sample size is very small. Mahmoudvand and Hassani (2009) proposed two new methods for constructing the confidence intervals for the CV of a normal distribution and compared them with Miller's, Makay's, Vangel's, and Sharma-Krishna's methods; they found that their proposed methods are more appropriate than the others. Buntao and Niwitpong (2012) proposed the generalized pivotal approach (GPA) and a closed-form method for variance estimation for the difference between the CVs of lognormal and delta-lognormal distributions; their results show that the GPA is the most suitable. After that, they constructed the confidence intervals for the ratio of the CVs of delta-lognormal distributions using GPA and the method of variance estimates recovery (MOVER) (Buntao and Niwitpong, 2013); their results were similar to the confidence intervals for the difference between the CVs. Wongkhao et al. (2015) presented the generalized confidence interval (GCI) and MOVER to construct the confidence intervals for the ratio of CVs of normal distributions and then compared their methods with the Verrill and Johnson and bootstrapping methods; they found that GCI and MOVER performed better than the others. Sangnawakij and Niwitpong (2017a) proposed MOVER, GCI, and the asymptotic confidence interval (ACI) for constructing the confidence interval for the $\mathrm{CV}$ and difference between the CVs of two-parameter exponential distributions; their results show that GCI was appropriate for a single CV and ACI worked well for the difference between the CVs. In addition, confidence intervals were extended by Sangnawakij and Niwitpong (2017b) based on the score and Wald intervals for the difference between and ratio of CVs of two gamma distributions; their proposed methods performed well in a comparative study. Recently, Yosboonruang et al. (2018) proposed GCI and a modified Fletcher method to construct the confidence intervals for the CV of a delta-lognormal distribution and found that GCI was the best. Afterward, they introduced the fiducial GCI (FGCI) and MOVER to construct the confidence intervals for the $\mathrm{CV}$ of a delta-lognormal distribution (Yosboonruang et al., 2019a). Moreover, they compared the confidence intervals based on FGCI and a Bayesian method 
Let $X_{i}=\left(X_{i 1}, X_{i 2}, \ldots, X_{i n_{i}}\right), i=1,2, \ldots, k$ be a random sample from $k$ independent delta-lognormal distributions, denoted by $X_{i j} \sim \Delta\left(\mu_{i}, \sigma_{i}^{2}, \delta_{i(0)}\right)$, where $\delta_{i(0)}=P\left(X_{i j}=0\right)$. Since this distribution contains zero and positive values, then the zero values follow a binomial distribution and the positive values a lognormal distribution denoted by $X_{i j}=0 \sim \operatorname{Bin}\left(n_{i}, \delta_{i(0)}\right)$ and $Y_{i j}=\ln \left(X_{i j}\right) \sim N\left(\mu_{i}, \sigma_{i}^{2}\right)$, respectively. Moreover, let $n_{i(0)}$ and $n_{i(1)}$ be the numbers of zero and positive values, respectively, where $n_{i}=n_{i(0)}+n_{i(1)}$. The distribution function of a delta-lognormal distribution is given by

$$
f\left(x_{i j} ; \mu_{i}, \sigma_{i}^{2}, \delta_{i(0)}\right)=\left\{\begin{array}{ll}
\delta_{i(0)} & ; x_{i j}=0 \\
\delta_{i(1)} \frac{1}{\sqrt{2 \pi} \sigma_{i}}\left(\frac{1}{x_{i j}}\right) \exp \left\{-\frac{\left[\ln \left(x_{i j}\right)-\mu_{i}\right]^{2}}{2 \sigma_{i}^{2}}\right\} & ; x_{i j}>0
\end{array},\right.
$$

where $\delta_{i(1)}=1-\delta_{i(0)}$. Following Aitchison (1955), the respective population mean and variance of $X_{i}$ are

$$
E\left(X_{i}\right)=\mu_{X_{i}}=\delta_{i(1)} \exp \left(\mu_{i}+\frac{\sigma_{i}^{2}}{2}\right)
$$


and

$$
\operatorname{Var}\left(X_{i}\right)=\sigma_{X_{i}}^{2}=\delta_{i(1)} \exp \left(2 \mu_{i}+\sigma_{i}^{2}\right)\left[\exp \left(\sigma_{i}^{2}\right)-\delta_{i(1)}\right] .
$$

Following this, the $\mathrm{CV}$ of $X_{i}$ can be expressed as

$$
C V\left(X_{i}\right)=v_{i}=\sqrt{\frac{\exp \left(\sigma_{i}^{2}\right)-\delta_{i(1)}}{\delta_{i(1)}}} .
$$

Since we are interested in constructing the SCIs for all pairwise differences between the CVs, then

$$
v_{i l}=v_{i}-v_{l}=\sqrt{\frac{\exp \left(\sigma_{i}^{2}\right)-\delta_{i(1)}}{\delta_{i(1)}}}-\sqrt{\frac{\exp \left(\sigma_{l}^{2}\right)-\delta_{l(1)}}{\delta_{l(1)}}},
$$

where $i, l=1,2, \ldots, k$ and $i \neq l$. The maximum likelihood estimators of $\delta_{i(1)}$ and $\mu_{i}$ are $\hat{\delta}_{i(1)}=n_{i(1)} / n_{i}$ and $\hat{\mu}_{i}=\sum_{j=1}^{n_{i(1)}} \ln \left(x_{i j}\right) / n_{i(1)}$, respectively. Furthermore, the unbiased estimator for $\sigma_{i}^{2}$ is $\hat{\sigma}_{i}^{2}=\sum_{j=1}^{n_{i(1)}}\left[\ln \left(x_{i j}\right)-\hat{\mu}_{i}\right]^{2} /\left(n_{i(1)}-1\right)$.

Assume that $\hat{\delta}_{i(1)}$ and $\hat{\sigma}_{i}^{2}$ are independent, then the maximum likelihood estimator of $v_{i}$ can be defined as

$$
\hat{v}_{i}=\sqrt{\frac{\exp \left(\hat{\sigma}_{i}^{2}\right)-\hat{\delta}_{i(1)}}{\hat{\delta}_{i(1)}}}
$$

Similarly,

$$
\hat{v}_{i l}=\hat{v}_{i}-\hat{v}_{l}=\sqrt{\frac{\exp \left(\hat{\sigma}_{i}^{2}\right)-\hat{\delta}_{i(1)}}{\hat{\delta}_{i(1)}}}-\sqrt{\frac{\exp \left(\hat{\sigma}_{l}^{2}\right)-\hat{\delta}_{l(1)}}{\hat{\delta}_{l(1)}}},
$$

where $i, l=1,2, \ldots, k$ and $i \neq l$.

According to Yosboonruang et al. (2020), the estimated variance of $\hat{v}_{i}-\hat{v}_{l}$ can be expressed as

$$
\begin{aligned}
\widehat{\operatorname{Var}}\left(\hat{v}_{i}-\hat{v}_{l}\right)= & \frac{\left\{\left[\ln \left(\hat{\delta}_{i(1)}\right)+\ln \left(\frac{\exp \left(\hat{\sigma}_{i}^{2}\right)-\hat{\delta}_{i(1)}}{\hat{\delta}_{i(1)}}+1\right)\right]\left[\frac{\exp \left(\hat{\sigma}_{i}^{2}\right)-\hat{\delta}_{i(1)}}{\hat{\delta}_{i(1)}}+1\right]\right\}^{2}}{2 n_{i}\left[\frac{\exp \left(\hat{\sigma}_{i}^{2}\right)-\hat{\delta}_{i(1)}}{\hat{\delta}_{i(1)}}\right]} \\
& +\frac{\left\{\left[\ln \left(\hat{\delta}_{l(1)}\right)+\ln \left(\frac{\exp \left(\hat{\sigma}_{l}^{2}\right)-\hat{\delta}_{l(1)}}{\hat{\delta}_{l(1)}}+1\right)\right]\left[\frac{\exp \left(\hat{\sigma}_{l}^{2}\right)-\hat{\delta}_{l(1)}}{\hat{\delta}_{l(1)}}+1\right]\right\}^{2}}{2 n_{l}\left[\frac{\exp \left(\hat{\sigma}_{l}^{2}\right)-\hat{\delta}_{l(1)}}{\hat{\delta}_{l(1)}}\right]},
\end{aligned}
$$

where $i, l=1,2, \ldots, k$ and $i \neq l$.

\section{The simultaneous FGCls}

To construct the simultaneous FGCIs, a fiducial generalized pivotal quantity (FGPQ), which is a subclass of the generalized pivotal quantity (GPQ) (Hannig et al., 2006), is presented as follows.

Definition 1. Let $X_{i}=\left(X_{i 1}, X_{i 2}, \ldots, X_{i n_{i}}\right), i=1,2, \ldots, k$ be a random sample from $k$ independent deltalognormal distributions with parameters of interest $\left(\sigma_{i}^{2}, \delta_{i(1)}\right)$ and nuisance parameter $\mu_{i}$. Let $x_{i}=$ $\left(x_{i 1}, x_{i 2}, \ldots, x_{i n_{i}}\right) i=1,2, \ldots, k$, be an observed value of $X_{i} . G P Q R\left(X_{i} ; x_{i}, \mu_{i}, \sigma_{i}^{2}, \delta_{i(1)}\right)$ is called an FGPQ if it corresponds with the following two conditions (Weerahandi, 1993; Hannig et al., 2006):

1. For a given $x_{i}$, the conditional distribution of $R\left(X_{i} ; x_{i}, \mu_{i}, \sigma_{i}^{2}, \delta_{i(1)}\right)$ is free of $\mu_{i}$.

2. The observed value of $R\left(X_{i} ; x_{i}, \mu_{i}, \sigma_{i}^{2}, \delta_{i(1)}\right)$ at $X_{i}=x_{i}, r\left(x_{i} ; x_{i}, \mu_{i}, \sigma_{i}^{2}, \delta_{i(1)}\right)$ is the parameter of interest. 
The FGPQs for $\sigma_{i}^{2}$ and $\delta_{i(1)}$ can be constructed by applying Definition 1. According to Hannig et al. (2006) and Li et al. (2013), the respective FGPQs for $\delta_{i(1)}$ and $\sigma_{i}^{2}$ are

$$
R_{\delta_{i(1)}} \sim \frac{1}{2} \operatorname{Beta}\left(n_{i(1)}, n_{i(0)}+1\right)+\frac{1}{2} \operatorname{Beta}\left(n_{i(1)}+1, n_{i(0)}\right)
$$

and

$$
R_{\sigma_{i}^{2}}=\frac{\left(n_{i(1)}-1\right) \hat{\sigma}_{i}^{2}}{U_{i}}
$$

where $U_{i} \sim \chi_{n_{i(1)}-1}^{2}$. Following this, the FGPQ for $v_{i}$ is simply

$$
R_{v_{i}}=\sqrt{\frac{\exp \left(R_{\sigma_{i}^{2}}\right)-R_{\delta_{i(1)}}}{R_{\delta_{i(1)}}}}
$$

Hence, the FGPQ for the differences between two independent CVs can be expressed as

$$
R_{v_{i l}}=R_{v_{i}}-R_{v_{l}}=\sqrt{\frac{\exp \left(R_{\sigma_{i}^{2}}\right)-R_{\delta_{i(1)}}}{R_{\delta_{i(1)}}}}-\sqrt{\frac{\exp \left(R_{\sigma_{l}^{2}}\right)-R_{\delta_{l(1)}}}{R_{\delta_{l(1)}}}}
$$

Accordingly,

$$
P\left[L_{i l} \leqslant v_{i}-v_{l} \leqslant U_{i l}, \forall i \neq l\right] \rightarrow 1-\alpha .
$$

This implies that

$$
P\left[R_{v_{i l}}(\alpha / 2) \leq R_{v_{i l}} \leq R_{v_{i l}}(1-\alpha / 2), \forall i \neq l\right] \rightarrow 1-\alpha .
$$


The Bayesian method

According to the distributions of $X_{i}$ for $i=1,2, \ldots, k$ with the unknown parameters $\mu_{i}, \sigma_{i}^{2}$, and $\delta_{i(0)}$, where $\delta_{i(0)}=1-\delta_{i(1)}$, the joint likelihood function of $k$ independent delta-lognormal distributions is

$$
L\left(\mu_{i}, \sigma_{i}^{2}, \delta_{i(1)} \mid x_{i j}\right) \propto \prod_{i=1}^{k}\left(1-\delta_{i(1)}\right)^{n_{i(0)}} \delta_{i(1)}^{n_{i(1)}}\left(\sigma_{i}^{2}\right)^{-\frac{n_{i(1)}}{2}} \exp \left\{-\frac{1}{2 \sigma_{i}^{2}} \sum_{j=1}^{n_{i(1)}}\left[\ln \left(x_{i j}\right)-\mu_{i}\right]^{2}\right\} .
$$

By applying the second-order partial derivative of the log-likelihood function with respect to the unknown parameters, the Fisher information matrix of the unknown parameters can be written as

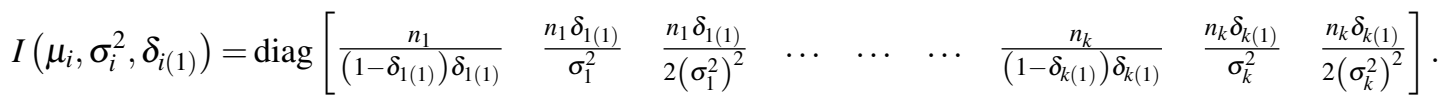

In this paper, we constructed both of equal-tailed SCIs based on simulation data and simultaneous credible intervals based on information from a simulation study of their prior distributions using two forms of Bayesian prior; the suitability of the Jeffreys' rule and uniform priors was determined by considering the values of a random variable of their posterior distributions that correspond to those for a delta-lognormal distribution. See also, Yosboonruang et al. (2019b) and Yosboonruang et al. (2020).

\section{The Jeffreys' rule prior}

The Jeffreys' rule prior is obtained from the square root of the determinant of the Fisher information matrix (Jeffreys, 1946). It is well known that a delta-lognormal distribution comprises lognormal and binomial distributions. From the CVs in Eq. (4), the parameters of interest are $\sigma_{i}^{2}$ and $\delta_{i(1)}$, and the Jeffreys' rule priors for these parameters are $p\left(\sigma_{i}^{2}\right) \propto \sigma_{i}^{-3}$ and $p\left(\delta_{i(1)}\right) \propto\left(1-\delta_{i(1)}\right)^{-\frac{1}{2}} \delta_{i(1)}^{\frac{1}{2}}$, respectively. Assuming that $\sigma_{i}^{2}$ and $\delta_{i(1)}$ are independent, the prior distribution for a delta-lognormal distribution can be defined as $p\left(\sigma_{i}^{2}, \delta_{i(1)}\right) \propto \sigma_{i}^{-3}\left(1-\delta_{i(1)}\right)^{-\frac{1}{2}} \delta_{i(1)}^{\frac{1}{2}}$. By combining the likelihood function and the prior distribution of a delta-lognormal distribution, the joint posterior density function can be written as

$$
\begin{aligned}
p\left(\sigma_{i}^{2}, \delta_{i(1)} \mid x_{i j}\right)= & \prod_{i=1}^{k} \frac{1}{\operatorname{Beta}\left(n_{i(0)}+\frac{1}{2}, n_{i(1)}+\frac{3}{2}\right)}\left(1-\delta_{i(1)}\right)^{\left(n_{i(0)}+\frac{1}{2}\right)-1} \delta_{i(1)}^{\left(n_{i(1)}+\frac{3}{2}\right)-1} \\
& \times \frac{1}{\sqrt{2 \pi} \frac{\sigma_{i}}{\sqrt{n_{i(1)}}}} \exp \left[-\frac{1}{2 \frac{\sigma_{i}^{2}}{n_{i(1)}}}\left(\mu_{i}-\hat{\mu}_{i}\right)^{2}\right] \frac{\left(\frac{n_{i(1)} \hat{\sigma}_{i}^{2}}{2}\right)^{\frac{n_{i(1)}}{2}}}{\Gamma\left(\frac{n_{i(1)}}{2}\right)}\left(\sigma_{i}^{2}\right)^{-\frac{n_{i(1)}}{2}-1} \exp \left(-\frac{\frac{n_{i(1)} \hat{\sigma}_{i}^{2}}{2}}{\sigma_{i}^{2}}\right),
\end{aligned}
$$

where $\hat{\mu}_{i}=\sum_{j=1}^{n_{i(1)}} \ln \left(x_{i j}\right) / n_{i(1)}$, and $\hat{\sigma}_{i}^{2}=\sum_{j=1}^{n_{i(1)}}\left[\ln \left(x_{i j}\right)-\hat{\mu}_{i}\right]^{2} /\left(n_{i(1)}-1\right)$.

By integrating Eq. (16), the respective posterior distributions of $\sigma_{i}^{2}$ and $\delta_{i(1)}$ are derived as

$$
p\left(\sigma_{i}^{2} \mid x_{i j}\right) \propto \prod_{i=1}^{k} \frac{\left(\frac{n_{i(1)} \hat{\sigma}_{i}^{2}}{2}\right)^{\frac{n_{i(1)}}{2}}}{\Gamma\left(\frac{n_{i(1)}}{2}\right)}\left(\sigma_{i}^{2}\right)^{-\frac{n_{i(1)}}{2}-1} \exp \left(-\frac{\frac{n_{i(1)} \hat{\sigma}_{i}^{2}}{2}}{\sigma_{i}^{2}}\right),
$$

and

$$
p\left(\delta_{i(1)} \mid x_{i j}\right) \propto \prod_{i=1}^{k} \frac{1}{\operatorname{Beta}\left(n_{i(0)}+\frac{1}{2}, n_{i(1)}+\frac{3}{2}\right)}\left(1-\delta_{i(1)}\right)^{\left(n_{i(0)}+\frac{1}{2}\right)-1} \delta_{i(1)}^{\left(n_{i(1)}+\frac{3}{2}\right)-1} .
$$

It should be noted that $p\left(\sigma_{i}^{2} \mid x_{i j}\right)$ follows an inverse gamma distribution and $p\left(\delta_{i(1)} \mid x_{i j}\right)$ follows a beta distribution, denoted by $\sigma_{i}^{2} \mid x_{i j} \sim \operatorname{Inv}-\operatorname{Gamma}\left(n_{i(1)} / 2, n_{i(1)} \hat{\sigma}_{i}^{2} / 2\right)$ and $\delta_{i(1)} \mid x_{i j} \sim \operatorname{Beta}\left(n_{i(0)}+1 / 2\right.$, $\left.n_{i(1)}+3 / 2\right)$, respectively. Consequently, $\sigma_{i}^{2} \mid x_{i j}$ and $\delta_{i(1)} \mid x_{i j}$ can be substituted into Eq. (5) to construct the equal-tailed SCI and the simultaneous credible interval, respectively. 


\section{The uniform prior}

Since the uniform prior has a constant function for the prior probability (Stone, 2013), then the uniform priors of $\sigma_{i}^{2}$ and $\delta_{i(1)}$ are 1 , denoted by $p\left(\sigma_{i}^{2}\right) \propto 1$ and $p\left(\delta_{i(1)}\right) \propto 1$, respectively. Afterward, the uniform prior for a delta-lognormal distribution becomes $p\left(\sigma_{i}^{2}, \delta_{i(1)}\right) \propto 1$. Similar to Eq. (16), the joint posterior density function is obtained by combining $p\left(\sigma_{i}^{2}, \delta_{i(1)}\right)$ with the likelihood function from Eq. (14). Subsequently, we obtain the posterior of $\sigma_{i}^{2}$ and $\delta_{i(1)}$ by integrating the joint posterior density function with respect to the others. Thus, the posterior distribution is $\sigma_{i}^{2} \mid x_{i j} \sim$ Inv-Gamma $\left[\left(n_{i(1)}-2\right) / 2,\left(n_{i(1)}-2\right) \hat{\sigma}_{i}^{2} / 2\right]$ for $\sigma_{i}^{2}$ and $\delta_{i(1)} \mid x_{i j} \sim \operatorname{Beta}\left(n_{i(0)}+1, n_{i(1)}+1\right)$ for $\delta_{i(1)}$.

Therefore, the $100(1-\alpha) \%$ equal-tailed SCI and simultaneous credible interval for $v_{i l}$ based on the Bayesian method are $L_{i l} \leq v_{i l} \leq U_{i l}$, where $L_{i l}$ and $U_{i l}$ are the lower and upper bounds of the intervals, respectively.

Theorem 2. Let $X_{i}=\left(X_{i 1}, X_{i 2}, \ldots, X_{i n_{i}}\right) \sim \Delta\left(\mu_{i}, \sigma_{i}^{2}, \delta_{i(1)}\right)$, where $i=1,2, \ldots, k$ and $\delta_{i(0)}=1-\delta_{i(1)}$, with sample sizes $n_{1}, n_{2}, \ldots, n_{k}$ and $n=n_{1}+n_{2}+\ldots+n_{k}$. Let $r_{i}=n_{i} / n$ as $n \rightarrow \infty$, where $0<r_{i}<1$. For $i, l=1,2, \ldots, k$ and $i \neq l$, let $v_{i}=\sqrt{\left[\exp \left(\sigma_{i}^{2}\right)-\delta_{i(1)}\right] / \delta_{i(1)}}$ and $v_{l}=\sqrt{\left[\exp \left(\sigma_{l}^{2}\right)-\delta_{l(1)}\right] / \delta_{l(1)}}$ be the $C V s$ of $X_{i}$ and $X_{l}$, respectively. Let $\hat{v}_{i}$ and $\hat{v}_{l}$ be the estimators of $v_{i}$ and $v_{l}$, respectively. An estimator for the variance of the difference between $v_{i}$ and $v_{l}$ is $\widehat{\operatorname{Var}}\left(\hat{v}_{i}-\hat{v}_{l}\right)$. Let $p\left(\sigma_{i}^{2}, \delta_{i(1)}\right)$ and $p\left(\sigma_{i}^{2}, \delta_{i(1)} \mid x_{i j}\right)$ be the prior distribution and the joint posterior density function for delta-lognormal distribution, respectively. Therefore,

$$
P\left[L_{i l} \leq v_{i l} \leq U_{i l}, \forall i \neq l\right] \rightarrow 1-\alpha
$$

Proof. The proof is similar to Theorem 1.

Algorithm 1: For the FGCI and Bayesian methods

Step 1. Generate random samples $X_{i}, i=1,2, \ldots, k$, with sample sizes $n_{1}, n_{2}, \ldots, n_{k}$ and calculate $\hat{\delta}_{i(1)}$ and $\hat{\sigma}_{i}^{2}$.

Step 2. Generate $U_{i} \sim \chi_{n_{i(1)}-1}^{2}$, Beta $\left(n_{i(1)}, n_{i(1)}+1\right)$, Beta $\left(n_{i(1)}+1, n_{i(1)}\right)$, $\operatorname{Beta}\left(n_{i(0)}+1 / 2, n_{i(1)}+3 / 2\right), \operatorname{Beta}\left(n_{i(0)}+1, n_{i(1)}+1\right), \operatorname{Inv}-\operatorname{Gamma}\left(n_{i(1)} / 2, n_{i(1)} \hat{\sigma}_{i}^{2} / 2\right)$, and Inv-Gamma $\left[\left(n_{i(1)}-2\right) / 2,\left(n_{i(1)}-2\right) \hat{\sigma}_{i}^{2} / 2\right]$.

Step 3. Calculate $R_{\delta_{i(1)}}, R_{\sigma_{i}^{2}}, R_{v_{i}}, R_{v_{l}}, v_{i}$, and $v_{l}$.

Step 4. Repeat Steps 2-3 5,000 times.

Step 5. Compute the $95 \%$ SCIs for vil.

Step 6. Repeat Steps 1-5 15,000 times.

\section{MOVER}

The concept of MOVER proposed by Donner and Zou (2012) can be applied to construct the $100(1-\alpha) \%$ two-sided confidence interval of $v_{i}-v_{l}$ for $i, l=1,2, \ldots, k$ and $i \neq l$, for which $L_{i l} \leq v_{i l} \leq U_{i l}$ where $L_{i l}$ and $U_{i l}$ denote the lower and upper limits of the confidence interval, respectively, expressed as

$$
L_{i l}=\hat{v}_{i}-\hat{v}_{l}-\sqrt{\left(\hat{v}_{i}-l_{i}\right)^{2}+\left(u_{l}-\hat{v}_{l}\right)^{2}}
$$

and

$$
U_{i l}=\hat{v}_{i}-\hat{v}_{l}+\sqrt{\left(u_{i}-\hat{v}_{i}\right)^{2}+\left(\hat{v}_{l}-l_{l}\right)^{2}}
$$

where $i, l=1,2, \ldots, k$ and $i \neq l$. From Eq. (4), the parameters of interest are $\delta_{i(1)}$ and $\sigma_{i}^{2}$, and so the confidence intervals for these parameters can be constructed. 
Since the unbiased estimator of $\sigma_{i}^{2}$ is given by $\hat{\sigma}_{i}^{2}=\sum_{j=1}^{n_{i(1)}}\left[\ln \left(x_{i j}\right)-\hat{\mu}_{i}\right]^{2} /\left(n_{i(1)}-1\right)$, for $i=1,2, \ldots, k$ and where $\left(n_{i(1)}-1\right) \hat{\sigma}_{i}^{2} / \sigma^{2} \sim \chi_{n_{i(1)}-1}^{2}$. Consequently, the respective lower and upper bounds for $\sigma_{i}^{2}$ are defined as

$$
l_{\sigma_{i}^{2}}=\frac{\left(n_{i(1)}-1\right) \hat{\sigma}_{i}^{2}}{\chi_{1-\frac{\alpha}{2}, n_{i(1)}-1}^{2}}
$$

and

$$
u_{\sigma_{i}^{2}}=\frac{\left(n_{i(1)}-1\right) \hat{\sigma}_{i}^{2}}{\chi_{\frac{\alpha}{2}, n_{i(1)}-1}^{2}}
$$

The score method proposed by Wilson (1927) is used to construct the confidence limits for $\delta_{i(1)}$. According to Brown et al. (2001) and Donner and Zou (2011), the respective lower and upper limits of $\delta_{i(1)}$ are given by

$$
l_{\delta_{i(1)}}=\frac{n_{i(1)}+\frac{Z_{i(\alpha / 2)}^{2}}{2}}{n_{i}+Z_{i(\alpha / 2)}^{2}}-Z_{i(\alpha / 2)} \frac{\sqrt{\frac{n_{i(0)} n_{i(1)}}{n_{i}}+\frac{Z_{i(\alpha / 2)}^{2}}{4}}}{n_{i}+Z_{i(\alpha / 2)}^{2}}
$$

and

$$
u_{\delta_{i(1)}}=\frac{n_{i(1)}+\frac{Z_{i(\alpha / 2)}^{2}}{2}}{n_{i}+Z_{i(\alpha / 2)}^{2}}+Z_{i(\alpha / 2)} \frac{\sqrt{\frac{n_{i(0)} n_{i(1)}}{n_{i}}+\frac{Z_{i(\alpha / 2)}^{2}}{4}}}{n_{i}+Z_{i(\alpha / 2)}^{2}},
$$

where $Z_{i}, i=1,2, \ldots, k$ follow a standard normal distribution. This approach is similar to constructing the confidence limits for $\sigma_{l}^{2}$ and $\delta_{l(1)}$.

Therefore, the $100(1-\alpha) \%$ two-sided SCIs for $v_{i}-v_{l}$ based on the MOVER method are

$$
S C I_{i l}=\left[L_{i l}, U_{i l}\right],
$$

where $i, l=1,2, \ldots, k$ and $i \neq l$.

Theorem 3. Let $X_{i}=\left(X_{i 1}, X_{i 2}, \ldots, X_{i n_{i}}\right), i=1,2, \ldots, k$, be random samples from $k$ independent deltalognormal distributions with mean $\mu_{i}$, variance $\sigma_{i}^{2}$, and probability of zero values $\delta_{i(0)}$. Furthermore, let the sample size of the $i^{\text {th }}$ random sample be $n_{i}$, where $n=n_{1}+n_{2}+\ldots+n_{k}$ and $r_{i}=n_{i} / n$ as $n \rightarrow \infty$, for which $0<r_{i}<1$. Let $v_{i}=\sqrt{\left[\exp \left(\sigma_{i}^{2}\right)-\delta_{i(1)}\right] / \delta_{i(1)}}$ and $v_{l}=\sqrt{\left[\exp \left(\sigma_{l}^{2}\right)-\delta_{l(1)}\right] / \delta_{l(1)}}$, for $i, l=1,2, \ldots, k$ and $i \neq l$, be the $C V s$ of $X_{i}$ and $X_{l}$, respectively. In addition, let $\hat{v}_{i}$ and $\hat{v}_{l}$ be the estimators of $v_{i}$ and $v_{l}$, respectively. Let $L_{i l}=\hat{v}_{i}-\hat{v}_{l}-\sqrt{\left(\hat{v}_{i}-l_{i}\right)^{2}+\left(u_{l}-\hat{v}_{l}\right)^{2}}$ and $U_{i l}=\hat{v}_{i}-\hat{v}_{l}+$ $\sqrt{\left(u_{i}-\hat{v}_{i}\right)^{2}+\left(\hat{v}_{l}-l_{l}\right)^{2}}$, where $i, l=1,2, \ldots, k$ and $i \neq l$, be the respective lower and upper limits of the confidence interval for $v_{i l}=v_{i}-v_{l}$. Therefore,

$$
P\left(L_{i l} \leq v_{i l} \leq U_{i l}, \forall i \neq l\right) \rightarrow 1-\alpha .
$$

Proof. Suppose that the respective lower and upper limits of the confidence interval for $v_{i l}=v_{i}-v_{l}$ are

$$
L_{i l}=\hat{v}_{i}-\hat{v}_{l}-\sqrt{\left(\hat{v}_{i}-l_{i}\right)^{2}+\left(u_{l}-\hat{v}_{l}\right)^{2}}=\hat{v}_{i l}-\sqrt{\left(\hat{v}_{i}-l_{i}\right)^{2}+\left(u_{l}-\hat{v}_{l}\right)^{2}}
$$

and

$$
U_{i l}=\hat{v}_{i}-\hat{v}_{l}+\sqrt{\left(u_{i}-\hat{v}_{i}\right)^{2}+\left(\hat{v}_{l}-l_{l}\right)^{2}}=\hat{v}_{i l}+\sqrt{\left(u_{i}-\hat{v}_{i}\right)^{2}+\left(\hat{v}_{l}-l_{l}\right)^{2}},
$$

where $i, l=1,2, \ldots, k$ and $i \neq l$. Thus, the respective estimators of variance for $\hat{v}_{i}$ and $\hat{v}_{l}$ at $v_{i}=l_{i}$ and $v_{l}=l_{l}$ are

$$
\widehat{\operatorname{Var}}\left(\hat{v}_{i}\right)=\frac{\left(\hat{v}_{i}-l_{i}\right)^{2}}{z_{\alpha / 2}^{2}}
$$


and

$$
\widehat{\operatorname{Var}}\left(\hat{v}_{l}\right)=\frac{\left(\hat{v}_{l}-l_{l}\right)^{2}}{z_{\alpha / 2}^{2}},
$$

where $z_{\alpha / 2}$ is the $\alpha / 2$-th quantile of the standard normal distribution. Similarly, the respective estimators of variance for $\hat{v}_{i}$ and $\hat{v}_{l}$ at $v_{i}=u_{i}$ and $v_{l}=u_{l}$ are

$$
\widehat{\operatorname{Var}}\left(\hat{v}_{i}\right)=\frac{\left(u_{i}-\hat{v}_{i}\right)^{2}}{z_{\alpha / 2}^{2}}
$$

and

$$
\widehat{\operatorname{Var}}\left(\hat{v}_{l}\right)=\frac{\left(u_{l}-\hat{v}_{l}\right)^{2}}{z_{\alpha / 2}^{2}} .
$$

Hence, the respective lower and upper limits can be expressed as

$$
\begin{aligned}
L_{i l} & =\hat{v}_{i l}-z_{\alpha / 2} \sqrt{\frac{\left(\hat{v}_{i}-l_{i}\right)^{2}}{z_{\alpha / 2}^{2}}+\frac{\left(u_{l}-\hat{v}_{l}\right)^{2}}{z_{\alpha / 2}^{2}}} \\
& =\hat{v}_{i l}-z_{\alpha / 2} \sqrt{\widehat{\operatorname{Var}}\left(\hat{v}_{i}\right)+\widehat{\operatorname{Var}}\left(\hat{v}_{l}\right)}
\end{aligned}
$$

and

$$
\begin{aligned}
U_{i l} & =\hat{v}_{i l}+z_{\alpha / 2} \sqrt{\frac{\left(u_{i}-\hat{v} i\right)^{2}}{z_{\alpha / 2}^{2}}+\frac{\left(\hat{v}_{l}-l_{l}\right)^{2}}{z_{\alpha / 2}^{2}}} \\
& =\hat{v}_{i l}+z_{\alpha / 2} \sqrt{\widehat{\operatorname{Var}}\left(\hat{v}_{i}\right)+\widehat{\operatorname{Var}}\left(\hat{v}_{l}\right)} .
\end{aligned}
$$

Therefore,

$$
\begin{aligned}
P\left(L_{i l} \leq v_{i l} \leq U_{i l}\right) & =P\left[v_{i l} \in\left(\hat{v}_{i l} \pm z_{\alpha / 2} \sqrt{\widehat{\operatorname{Var}}\left(\hat{v}_{i}\right)+\widehat{\operatorname{Var}}\left(\hat{v}_{l}\right)}\right), \forall i \neq l\right] \\
& =P\left[\max _{i \neq l}\left|\frac{\hat{v}_{i l}-v_{i l}}{\sqrt{\widehat{\operatorname{Var}}\left(\hat{v}_{i}\right)+\widehat{\operatorname{Var}}\left(\hat{v}_{l}\right)}}\right| \leq z_{\alpha / 2}\right] \\
& =P\left[D_{n}^{\prime} \leq z_{\alpha / 2}\right] .
\end{aligned}
$$

Suppose that $n_{i} / n \rightarrow r_{i} \in(0,1)$ as $n \rightarrow \infty, i=1,2, \ldots, k$ where $n=n_{1}+n_{2}+\ldots+n_{k}$. From the central limit theorem, $n\left(\hat{v}_{i}-v_{i}\right) \stackrel{d}{\rightarrow} Z_{i}, i=1,2, \ldots, k$, where $Z_{i} \stackrel{i i d}{\sim} N\left(0, \sigma_{i}^{2} / r_{i}\right)$, while from Slutsky's theorem, $D_{n}^{\prime} \rightarrow D^{\prime}$, where $D^{\prime}=\max _{i \neq l}\left|\left(Z_{i}-Z_{l}\right) / \sqrt{\sigma_{i}^{2} / r_{i}+\sigma_{l}^{2} / r_{l}}\right|$.

Following Skorokhod's theorem, let $Y_{n}$ and $Y$ be random variables from the common probability space with distributions $D_{n}^{\prime}$ and $D^{\prime}$, respectively. Thus, $Y_{n}$ converges to $Y$ almost surely, denoted by $Y_{n} \stackrel{a . s .}{\rightarrow} Y$, and $D_{n}^{\prime}$ converges to $D^{\prime}$ almost surely, denoted by $D_{n}^{\prime} \stackrel{\text { a.s. }}{\rightarrow} D^{\prime}$. Assume that $Z_{i}$ and $Z_{i}^{*}$ are independent and identically distributed random variables. Thus, $T\left(X, X^{*}, \mu, \sigma^{2}\right) \rightarrow D^{*}$, where $D^{\prime *}=$ $\max _{i \neq l}\left|\left(Z_{i}^{*}-Z_{l}^{*} / \sqrt{\sigma_{i}^{2} / r_{i}+\sigma_{l}^{2} / r_{l}}\right)\right|$, for $i, l=1,2, \ldots, k$, and $i \neq l$.

Since the limiting distribution of $T\left(X, X^{*}, \mu, \sigma^{2}\right)$ is continuous and $z_{\alpha / 2}(X) \rightarrow q_{\alpha / 2}$, where $q_{\alpha / 2}$ is the $\alpha / 2$-th quantile of the distribution of $D^{\prime *}$, we can obtain

$$
P\left(D_{n}^{\prime} \leq z_{\alpha / 2}\right) \rightarrow P\left(D^{\prime} \leq q_{\alpha / 2}\right)=P\left(D^{\prime *} \leq q_{\alpha / 2}\right)=1-\alpha,
$$

as $n \rightarrow \infty$. Therefore,

$$
P\left[v_{i l} \in\left(\hat{v}_{i l} \pm z_{\alpha / 2} \sqrt{\widehat{\operatorname{Var}}\left(\hat{v}_{i}\right)+\widehat{\operatorname{Var}}\left(\hat{v}_{l}\right)}, \forall i \neq l\right)\right] \rightarrow 1-\alpha,
$$


which implies that

$$
P\left(L_{i l} \leq v_{i l} \leq U_{i l}, \forall i \neq l\right) \rightarrow 1-\alpha .
$$

199

Algorithm 2: For MOVER

Step 1. Generate random samples $X_{i}, i=1,2, \ldots, k$ with sample size $n_{1}, n_{2}, \ldots, n_{k}$ and calculate $\hat{\delta}_{i(1)}$ and $\hat{\sigma}_{i}^{2}$.

Step 2. Generate $\chi_{1-\alpha / 2, n_{i(1)}-1}^{2}, \chi_{\alpha / 2, n_{i(1)}-1}^{2}$, and $Z_{i} \sim N(0,1)$.

Step 3. Calculate $l_{\sigma_{i}^{2}}, l_{\sigma_{l}^{2}}, u_{\sigma_{i}^{2}}, u_{\sigma_{l}^{2}}, l_{\delta_{i(1)}}, l_{\delta_{l(1)}}, u_{\delta_{i(1)}}$, and $u_{\delta_{l(1)}}$.

Step 4. Repeat Steps 2-3 5,000 times.

Step 5. Compute the $95 \%$ SCIs for $v_{i l}$.

Step 6. Repeat Steps 1-5 15,000 times.

\section{SIMULATION RESULTS}

Here, the performances of the proposed methods via Monte Carlo simulation with the R statistical program are presented. The best method attains a coverage probability equal to or greater than the nominal simultaneous confidence level of 0.95 together with the shortest expected length. The simulations were conducted with 15,000 iterations for each combination of parameters. Furthermore, 5,000 replications for the FGCI and Bayesian methods for each case of parameter combination were carried out. Sample sizes were set as 25,50 , and $100 ; \delta_{i(1)}=0.2,0.5,0.8$; and $\sigma_{i}^{2}=0.5,1.0,2.0$.

The results in Tables 2 - 4 and Figs. 1 - 3 show that the coverage probabilities of FGCI and the equal-tailed Bayesian using Jeffreys' rule prior were close to or greater than the nominal confidence level for almost all $k$ values. Similarly, the coverage probabilities of the equal-tailed Bayesian using the uniform prior, the Bayesian credible intervals using Jeffreys' rule and uniform priors, and MOVER were close to or greater than the nominal confidence level for all cases. For most cases, the Bayesian credible interval using Jeffreys' rule prior attained the shortest expected length, except for $n_{i}=50 ; \delta_{i(1)}=0.5,0.8$; and $\sigma_{i}^{2}=0.5,1.0$, for which the expected lengths of FGCI were the shortest.

\section{EMPIRICAL STUDY}

Thailand is generally divided into five areas by topography, i.e. Northern (A1), Northeastern (A2), Central (A3), Eastern (A4), and Southern (A5). The daily rainfall data from these areas in August 2020 were used to assess the performances of the proposed methods for SCI construction. The distributions of these data are presented in Fig. 4, which shows right-skewness for all of the datasets. Thus, the minimum Akaike information criterion (AIC) and the lowest Bayesian information criterion (BIC) were used to test the fitting of the distributions to such data. From AIC and BIC results in Table 5, it is evident that the positive values in the rainfall datasets from the five areas conform to lognormal distributions. Moreover, normal Q-Q plots were constructed to show the distributions of the log-transformed positive rainfall data from the five areas (Fig. 5), which verified the AIC and BIC results that these datasets follow lognormal distributions. A summary of these data are

$$
\begin{aligned}
& n_{1}=31, \hat{\delta}_{1}=0.7097, \hat{\mu}_{1}=0.7715, \hat{\sigma}_{1}^{2}=3.4565, \hat{\eta}_{1}=6.6088, \\
& n_{2}=31, \hat{\delta}_{2}=0.6774, \hat{\mu}_{2}=1.4332, \hat{\sigma}_{2}^{2}=2.9550, \hat{\eta}_{1}=5.2294, \\
& n_{3}=31, \hat{\delta}_{3}=0.6452, \hat{\mu}_{3}=1.5512, \hat{\sigma}_{3}^{2}=2.8638, \hat{\eta}_{3}=5.1154, \\
& n_{4}=31, \hat{\delta}_{4}=0.4839, \hat{\mu}_{4}=1.4178, \hat{\sigma}_{4}^{2}=2.1487, \hat{\eta}_{4}=4.0888, \\
& n_{5}=31, \hat{\delta}_{5}=0.4839, \hat{\mu}_{5}=1.8040, \hat{\sigma}_{5}^{2}=2.1962, \hat{\eta}_{5}=4.1930 .
\end{aligned}
$$

Table 6 reports the $95 \%$ SCIs and credible intervals for all pairwise differences between the CVs of the daily rainfall series from five areas in Thailand. The results show that the expected length of the Bayesian credible interval using the Jeffreys' rule prior was the shortest, which corresponds with the simulation results. Therefore, it is a good choice for constructing the SCI for all of the pairwise differences between the CVs of the precipitation series from the five areas in Thailand. 
Table 2. The coverage probabilities and expected lengths for the $95 \%$ SCIs and credible intervals for all pairwise differences between the CVs of delta-lognormal distributions for $k=3$.

\begin{tabular}{|c|c|c|c|c|c|c|c|c|}
\hline \multirow{2}{*}{$n_{1}: n_{2}: n_{3}$} & \multirow{2}{*}{$\delta_{1(1)}: \delta_{2(1)}: \delta_{3(1)}$} & \multirow{2}{*}{$\sigma_{1}^{2}: \sigma_{2}^{2}: \sigma_{3}^{2}$} & \multicolumn{6}{|c|}{ Coverage probabilities (Expected lengths) } \\
\hline & & & FGCI & B.Jrule-E & B.Uni-E & B.Jrule-C & B.Uni-C & MOVER \\
\hline \multirow[t]{16}{*}{$25: 25: 25$} & \multirow[t]{8}{*}{$0.5: 0.5: 0.5$} & \multirow[t]{2}{*}{$0.5: 0.5: 0.5$} & 0.9642 & 0.9788 & 0.9842 & 0.9956 & 0.9980 & 0.9986 \\
\hline & & & $(2.1698)$ & $(2.1537)$ & $(2.5030)$ & (2.0848) & $(2.4026)$ & $(3.4408)$ \\
\hline & & \multirow[t]{2}{*}{$1.0: 1.0: 1.0$} & 0.9573 & 0.9605 & 0.9718 & 0.9957 & 0.9984 & 0.9941 \\
\hline & & & $(6.8818)$ & $(6.2442)$ & $(7.9188)$ & $(5.7177)$ & $(7.1021)$ & $(9.6034)$ \\
\hline & & $2.0: 2.0: 2.0$ & 0.9516 & 0.9465 & 0.9631 & 0.9978 & 0.9991 & 0.9838 \\
\hline & & & $(68.0142)$ & $(53.9752)$ & $(88.1169)$ & (37.9389) & $(55.1280)$ & $(85.1444)$ \\
\hline & & $0.5: 1.0: 2.0$ & 0.9557 & 0.9540 & 0.9678 & 0.9708 & 0.9820 & 0.9878 \\
\hline & & & $(24.7584)$ & (20.5209) & (31.5389) & (13.1644) & $(17.7557)$ & $(31.4612)$ \\
\hline & $0.8: 0.8: 0.8$ & $0.5: 0.5: 0.5$ & 0.9542 & 0.9632 & 0.9725 & 0.9834 & 0.9894 & 0.9954 \\
\hline & & & (1.2558) & (1.2613) & (1.3684) & (1.2412) & (1.3444) & (1.8437) \\
\hline & & $1.0: 1.0: 1.0$ & 0.9533 & 0.9526 & 0.9636 & 0.9857 & 0.9915 & 0.9868 \\
\hline & & & (3.2363) & (3.1117) & $(3.4638)$ & (2.9940) & $(3.3202)$ & $(4.2113)$ \\
\hline & & $2.0: 2.0: 2.0$ & 0.9499 & 0.9458 & 0.9570 & 0.9930 & 0.9963 & 0.9756 \\
\hline & & & (16.6440) & (15.4579) & (18.2078) & (13.7933) & (16.0213) & (20.0584) \\
\hline & & $0.5: 1.0: 2.0$ & 0.9513 & 0.9496 & 0.9598 & 0.9659 & 0.9750 & 0.9797 \\
\hline & & & (7.3206) & (6.8777) & (7.9629) & (5.5921) & (6.3072) & $(8.7800)$ \\
\hline $50: 50: 50$ & $0.2: 0.2: 0.2$ & $0.5: 0.5: 0.5$ & 0.9692 & 0.9869 & 0.9906 & 0.9991 & 0.9997 & 0.9994 \\
\hline & & & $(4.3383)$ & (4.1487) & $(5.2706)$ & (3.9321) & $(4.8731)$ & (7.2083) \\
\hline & & $1.0: 1.0: 1.0$ & 0.9593 & 0.9672 & 0.9778 & 0.9988 & 0.9996 & 0.9965 \\
\hline & & & $(17.7495)$ & (14.6338) & (23.0519) & $(12.3745)$ & (17.8689) & $(25.8720)$ \\
\hline & & $2.0: 2.0: 2.0$ & 0.9525 & 0.9489 & 0.9668 & 0.9984 & 0.9996 & 0.9882 \\
\hline & & & (813.5319) & (360.7209) & $(3.75 \mathrm{E}+03)$ & (130.6376) & (338.9741) & $(1.00 \mathrm{E}+03)$ \\
\hline & & $0.5: 1.0: 2.0$ & 0.9560 & 0.9567 & 0.9719 & 0.9742 & 0.9846 & 0.9910 \\
\hline & & & (131.2015) & (80.4363) & (238.4643) & (36.1680) & $(67.2572)$ & $(169.2385)$ \\
\hline & $0.5: 0.5: 0.5$ & $0.5: 0.5: 0.5$ & 0.9609 & 0.9797 & 0.9827 & 0.9904 & 0.9926 & 0.9990 \\
\hline & & & (1.2086) & (1.2989) & (1.3657) & (1.2849) & (1.3499) & (1.9562) \\
\hline & & $1.0: 1.0: 1.0$ & 0.9536 & 0.9613 & 0.9678 & 0.9870 & 0.9907 & 0.9934 \\
\hline & & & $(3.0015)$ & $(2.9770)$ & (3.1957) & (2.8997) & (3.1064) & $(4.2360)$ \\
\hline & & $2.0: 2.0: 2.0$ & 0.9496 & 0.9488 & 0.9579 & 0.9910 & 0.9942 & 0.9831 \\
\hline & & & (13.0784) & (12.5308) & (13.8315) & (11.6629) & (12.8048) & (16.6567) \\
\hline & & $0.5: 1.0: 2.0$ & 0.9510 & 0.9541 & 0.9619 & 0.9644 & 0.9712 & 0.9872 \\
\hline & & & $(6.2117)$ & $(6.0214)$ & $(6.5869)$ & $(5.1934)$ & $(5.6079)$ & (7.9402) \\
\hline & $0.8: 0.8: 0.8$ & $0.5: 0.5: 0.5$ & 0.9545 & 0.9652 & 0.9702 & 0.9764 & 0.9804 & 0.9960 \\
\hline & & & $(0.7563)$ & $(0.7866)$ & $(0.8128)$ & $(0.7810)$ & $(0.8069)$ & $(1.1146)$ \\
\hline & & $1.0: 1.0: 1.0$ & 0.9512 & 0.9530 & 0.9582 & 0.9744 & 0.9788 & 0.9870 \\
\hline & & & $(1.7222)$ & (1.7159) & (1.7838) & (1.6925) & (1.7587) & $(2.2244)$ \\
\hline & & $2.0: 2.0: 2.0$ & 0.9489 & 0.9476 & 0.9528 & 0.9832 & 0.9863 & 0.9746 \\
\hline & & & $(6.2458)$ & $(6.1250)$ & $(6.4310)$ & (5.9199) & (6.2078) & (7.4204) \\
\hline & & $0.5: 1.0: 2.0$ & 0.9534 & 0.9532 & 0.9592 & 0.9644 & 0.9690 & 0.9816 \\
\hline & & & (3.2059) & $(3.1646)$ & (3.3108) & (2.8957) & (3.0156) & (3.8254) \\
\hline $100: 100: 100$ & $0.2: 0.2: 0.2$ & $0.5: 0.5: 0.5$ & 0.9655 & 0.9862 & 0.9876 & 0.9955 & 0.9969 & 0.9995 \\
\hline & & & (2.1032) & $(2.2970)$ & $(2.4455)$ & (2.2649) & $(2.4065)$ & $(3.5694)$ \\
\hline & & $1.0: 1.0: 1.0$ & 0.9572 & 0.9696 & 0.9746 & 0.9935 & 0.9959 & 0.9966 \\
\hline & & & $(5.5368)$ & $(5.4606)$ & $(6.0091)$ & (5.2547) & $(5.7570)$ & $(8.2473)$ \\
\hline & & $2.0: 2.0: 2.0$ & 0.9528 & 0.9546 & 0.9629 & 0.9951 & 0.9973 & 0.9891 \\
\hline & & & (28.1449) & $(26.2788)$ & $(30.4653)$ & (23.6063) & $(27.0268)$ & (37.4768) \\
\hline & & $0.5: 1.0: 2.0$ & 0.9541 & 0.9585 & 0.9665 & 0.9672 & 0.9744 & 0.9903 \\
\hline & & & (12.6988) & (12.0766) & (13.7614) & (9.9027) & (11.0231) & (16.9562) \\
\hline & $0.5: 0.5: 0.5$ & $0.5: 0.5: 0.5$ & 0.9595 & 0.9793 & 0.9803 & 0.9852 & 0.9866 & 0.9988 \\
\hline & & & $(0.7728)$ & $(0.8596)$ & $(0.8774)$ & $(0.8547)$ & $(0.8723)$ & $(1.2568)$ \\
\hline & & $1.0: 1.0: 1.0$ & 0.9558 & 0.9646 & 0.9673 & 0.9806 & 0.9824 & 0.9949 \\
\hline & & & (1.7356) & (1.7761) & $(1.8252)$ & $(1.7576)$ & (1.8058) & $(2.4523)$ \\
\hline & & $2.0: 2.0: 2.0$ & 0.9501 & 0.9513 & 0.9555 & 0.9803 & 0.9831 & 0.9832 \\
\hline & & & $(6.1101)$ & $(6.0586)$ & $(6.2714)$ & (5.9140) & (6.1174) & $(7.6906)$ \\
\hline & & $0.5: 1.0: 2.0$ & 0.9518 & 0.9565 & 0.9603 & 0.9613 & 0.9653 & 0.9889 \\
\hline & & & (3.1854) & (3.1976) & (3.2977) & (2.9846) & (3.0716) & $(4.0742)$ \\
\hline & $0.8: 0.8: 0.8$ & $0.5: 0.5: 0.5$ & 0.9542 & 0.9668 & 0.9691 & 0.9721 & 0.9739 & 0.9966 \\
\hline & & & $(0.5013)$ & $(0.5298)$ & $(0.5376)$ & $(0.5273)$ & $(0.5350)$ & $(0.7395)$ \\
\hline & & $1.0: 1.0: 1.0$ & 0.9495 & 0.9526 & 0.9554 & 0.9654 & 0.9678 & 0.9864 \\
\hline & & & (1.0816) & (1.0919) & (1.1103) & (1.0843) & $(1.1025)$ & (1.3892) \\
\hline & & $2.0: 2.0: 2.0$ & 0.9517 & 0.9516 & 0.9546 & 0.9739 & 0.9756 & 0.9766 \\
\hline & & & (3.4703) & $(3.4545)$ & $(3.5212)$ & (3.4088) & $(3.4741)$ & $(4.0656)$ \\
\hline & & $0.5: 1.0: 2.0$ & 0.9496 & 0.9518 & 0.9552 & 0.9579 & 0.9608 & 0.9814 \\
\hline & & & (1.8867) & (1.8883) & (1.9231) & (1.8062) & (1.8377) & $(2.2430)$ \\
\hline $25: 50: 100$ & $0.5: 0.5: 0.5$ & $0.5: 0.5: 0.5$ & 0.9614 & 0.9782 & 0.9840 & 0.9829 & 0.9885 & 0.9984 \\
\hline & & & (1.4118) & (1.4647) & (1.6172) & (1.4069) & (1.5307) & $(2.2374)$ \\
\hline & & $1.0: 1.0: 1.0$ & 0.9525 & 0.9572 & 0.9670 & 0.9764 & 0.9838 & 0.9941 \\
\hline & & & (3.9253) & $(3.7120)$ & $(4.3712)$ & (3.3455) & (3.7992) & $(5.4323)$ \\
\hline & & $2.0: 2.0: 2.0$ & 0.9525 & 0.9502 & 0.9609 & 0.9827 & 0.9875 & 0.9826 \\
\hline & & & $(27.5125)$ & $(23.2226)$ & (33.9099) & (16.5621) & $(20.9132)$ & (34.6995) \\
\hline & & $0.5: 1.0: 2.0$ & 0.9551 & 0.9600 & 0.9648 & 0.9793 & 0.9827 & 0.9901 \\
\hline & & & (3.8830) & (3.8512) & (4.0951) & (3.6913) & (3.9292) & (5.2078) \\
\hline
\end{tabular}


Table 2. (Continued).

\begin{tabular}{|c|c|c|c|c|c|c|c|c|}
\hline \multirow[b]{2}{*}{$n_{1}: n_{2}: n_{3}$} & \multirow{2}{*}{$\delta_{1(1)}: \delta_{2(1)}: \delta_{3(1)}$} & \multirow{2}{*}{$\sigma_{1}^{2}: \sigma_{2}^{2}: \sigma_{3}^{2}$} & \multicolumn{6}{|c|}{ Coverage probabilities (Expected lengths) } \\
\hline & & & FGCI & B.Jrule-E & B.Uni-E & B.Jrule-C & B.Uni-C & MOVER \\
\hline \multirow[t]{8}{*}{$25: 50: 100$} & $0.8: 0.8: 0.8$ & $0.5: 0.5: 0.5$ & 0.9572 & 0.9679 & 0.9740 & 0.9754 & 0.9825 & 0.9958 \\
\hline & & & $(0.8600)$ & $(0.8810)$ & $(0.9323)$ & $(0.8589)$ & $(0.9050)$ & (1.2513) \\
\hline & & $1.0: 1.0: 1.0$ & 0.9516 & 0.9525 & 0.9596 & 0.9709 & 0.9775 & 0.9869 \\
\hline & & & $(2.0776)$ & $(2.0320)$ & (2.1907) & (1.9232) & $(2.0528)$ & $(2.6582)$ \\
\hline & & $2.0: 2.0: 2.0$ & 0.9513 & 0.9486 & 0.9558 & 0.9776 & 0.9826 & 0.9751 \\
\hline & & & $(8.8667)$ & (8.4294) & $(9.4811)$ & (7.3245) & $(8.0262)$ & $(10.4756)$ \\
\hline & & $0.5: 1.0: 2.0$ & 0.9532 & 0.9545 & 0.9592 & 0.9702 & 0.9735 & 0.9833 \\
\hline & & & $(2.2424)$ & $(2.2348)$ & $(2.3100)$ & (2.1757) & (2.2505) & $(2.7807)$ \\
\hline
\end{tabular}

Note: B.Jrule-E, B.Uni-E represented the equal-tailed Bayesian confidence intervals using Jeffreys' rule and uniform priors, respectively, and B.Jrule-C and B.Uni-C represented the Bayesian credible intervals using Jeffrey's rule and uniform priors.

Table 3. The coverage probabilities and expected lengths for the $95 \%$ SCIs and credible intervals for all pairwise differences between the CVs of delta-lognormal distributions for $k=5$.

\begin{tabular}{|c|c|c|c|c|c|c|c|c|}
\hline \multirow{2}{*}{$n_{1}: \ldots: n_{5}$} & \multirow{2}{*}{$\delta_{1(1)}: \ldots: \delta_{5(1)}$} & \multirow{2}{*}{$\sigma_{1}^{2}: \ldots: \sigma_{5}^{2}$} & \multicolumn{6}{|c|}{ Coverage probabilities (Expected lengths) } \\
\hline & & & FGCI & B.Jrule-E & B.Uni-E & B.Jrule-C & B.Uni-C & MOVER \\
\hline \multirow[t]{16}{*}{$25^{5}$} & \multirow[t]{8}{*}{$0.5^{5}$} & \multirow[t]{2}{*}{$0.5^{5}$} & 0.9643 & 0.9794 & 0.9854 & 0.9958 & 0.9981 & 0.9986 \\
\hline & & & $(2.1555)$ & $(2.1428)$ & $(2.4893)$ & (2.0756) & $(2.3915)$ & $(3.4245)$ \\
\hline & & \multirow[t]{2}{*}{$1.0^{5}$} & 0.9558 & 0.9597 & 0.9717 & 0.9954 & 0.9982 & 0.9935 \\
\hline & & & $(6.9019)$ & $(6.2584)$ & (7.9374) & $(5.7313)$ & $(7.1150)$ & $(9.6234)$ \\
\hline & & \multirow[t]{2}{*}{$2.0^{5}$} & 0.9519 & 0.9470 & 0.9625 & 0.9973 & 0.9993 & 0.9834 \\
\hline & & & $(62.1565)$ & $(50.5751)$ & $(80.8805)$ & $(37.1890)$ & (53.9109) & (79.5531) \\
\hline & & \multirow[t]{2}{*}{$0.5^{2}: 1.0: 2.0^{2}$} & 0.9545 & 0.9538 & 0.9676 & 0.9715 & 0.9818 & 0.9870 \\
\hline & & & $(25.5380)$ & (23.5178) & (37.1670) & (15.2344) & (20.9148) & $(36.4956)$ \\
\hline & \multirow[t]{8}{*}{$0.8^{5}$} & \multirow[t]{2}{*}{$0.5^{5}$} & 0.9548 & 0.9642 & 0.9728 & 0.9833 & 0.9895 & 0.9958 \\
\hline & & & $(1.2560)$ & $(1.2612)$ & $(1.3683)$ & (1.2413) & $(1.3445)$ & (1.8432) \\
\hline & & \multirow[t]{2}{*}{$1.0^{5}$} & 0.9529 & 0.9523 & 0.9627 & 0.9857 & 0.9915 & 0.9875 \\
\hline & & & $(3.2430)$ & (3.1186) & $(3.4726)$ & (3.0017) & $(3.3297)$ & $(4.2211)$ \\
\hline & & \multirow[t]{2}{*}{$2.0^{5}$} & 0.9495 & 0.9447 & 0.9567 & 0.9929 & 0.9963 & 0.9757 \\
\hline & & & (16.3407) & (15.2264) & $(17.9101)$ & (13.5908) & $(15.7761)$ & (19.7367) \\
\hline & & \multirow[t]{2}{*}{$0.5^{2}: 1.0: 2.0^{2}$} & 0.9525 & 0.9508 & 0.9614 & 0.9696 & 0.9775 & 0.9807 \\
\hline & & & $(7.2308)$ & $(7.5393)$ & $(8.7262)$ & (6.1994) & (7.0193) & $(9.6191)$ \\
\hline \multirow[t]{24}{*}{$50^{5}$} & $0.2^{5}$ & $0.5^{5}$ & 0.9676 & 0.9864 & 0.9896 & 0.9990 & 0.9996 & 0.9994 \\
\hline & & & $(4.3044)$ & $(4.1252)$ & $(5.2361)$ & (3.9094) & $(4.8405)$ & $(7.1615)$ \\
\hline & & $1.0^{5}$ & 0.9610 & 0.9688 & 0.9792 & 0.9988 & 0.9997 & 0.9969 \\
\hline & & & (17.6359) & (14.5147) & (22.9054) & (12.3017) & (17.7946) & $(25.7023)$ \\
\hline & & $2.0^{5}$ & 0.9532 & 0.9499 & 0.9673 & 0.9988 & 0.9997 & 0.9882 \\
\hline & & & $(428.000)$ & (253.198) & (996.537) & (122.265) & $(272.000)$ & (578.397) \\
\hline & & $0.5^{2}: 1.0: 2.0^{2}$ & 0.9548 & 0.9550 & 0.9707 & 0.9735 & 0.9849 & 0.9903 \\
\hline & & & (191.069) & $(122.152)$ & (490.477) & (47.626) & (101.477) & (276.993) \\
\hline & $0.5^{5}$ & $0.5^{5}$ & 0.9602 & 0.9789 & 0.9818 & 0.9899 & 0.9921 & 0.9987 \\
\hline & & & (1.2089) & $(1.2989)$ & $(1.3660)$ & $(1.2850)$ & (1.3502) & (1.9561) \\
\hline & & $1.0^{5}$ & 0.9548 & 0.9618 & 0.9677 & 0.9876 & 0.9911 & 0.9941 \\
\hline & & & $(3.0083)$ & $(2.9852)$ & $(3.2034)$ & (2.9072) & $(3.1133)$ & $(4.2457)$ \\
\hline & & $2.0^{5}$ & 0.9506 & 0.9496 & 0.9578 & 0.9913 & 0.9944 & 0.9839 \\
\hline & & & (13.0787) & (12.5396) & (13.8536) & (11.6771) & (12.8287) & (16.6737) \\
\hline & & $0.5^{2}: 1.0: 2.0^{2}$ & 0.9537 & 0.9574 & 0.9644 & 0.9695 & 0.9752 & 0.9881 \\
\hline & & & $(6.1486)$ & $(6.5705)$ & $(7.1952)$ & $(5.7079)$ & $(6.1752)$ & $(8.6478)$ \\
\hline & $0.8^{5}$ & $0.5^{5}$ & 0.9541 & 0.9654 & 0.9700 & 0.9763 & 0.9799 & 0.9958 \\
\hline & & & $(0.7579)$ & $(0.7881)$ & $(0.8142)$ & $(0.7825)$ & $(0.8083)$ & (1.1163) \\
\hline & & $1.0^{5}$ & 0.9516 & 0.9536 & 0.9589 & 0.9755 & 0.9790 & 0.9874 \\
\hline & & & $(1.7233)$ & $(1.7170)$ & $(1.7855)$ & (1.6937) & $(1.7603)$ & $(2.2261)$ \\
\hline & & $2.0^{5}$ & 0.9508 & 0.9495 & 0.9548 & 0.9838 & 0.9871 & 0.9760 \\
\hline & & & $(6.2622)$ & $(6.1471)$ & $(6.4545)$ & $(5.9443)$ & $(6.2338)$ & $(7.4475)$ \\
\hline & & $0.5^{2}: 1.0: 2.0^{2}$ & 0.9512 & 0.9522 & 0.9574 & 0.9646 & 0.9691 & 0.9808 \\
\hline & & & (3.1366) & $(3.3900)$ & $(3.5463)$ & (3.1199) & $(3.2508)$ & $(4.0931)$ \\
\hline $100^{5}$ & $0.2^{5}$ & $0.5^{5}$ & 0.9660 & 0.9867 & 0.9883 & 0.9954 & 0.9966 & 0.9994 \\
\hline & & & (2.1027) & $(2.2959)$ & $(2.4445)$ & (2.2638) & $(2.4055)$ & $(3.5676)$ \\
\hline & & $1.0^{5}$ & 0.9566 & 0.9680 & 0.9732 & 0.9935 & 0.9958 & 0.9968 \\
\hline & & & $(5.5583)$ & $(5.4771)$ & $(6.0303)$ & (5.2701) & (5.7768) & $(8.2755)$ \\
\hline & & $2.0^{5}$ & 0.9521 & 0.9526 & 0.9617 & 0.9951 & 0.9971 & 0.9883 \\
\hline & & & $(27.9967)$ & (26.1432) & $(30.2853)$ & (23.4497) & (26.8163) & $(37.2629)$ \\
\hline & & $0.5^{2}: 1.0: 2.0^{2}$ & 0.9550 & 0.9598 & 0.9676 & 0.9697 & 0.9763 & 0.9915 \\
\hline & & & $(12.6571)$ & $(13.2932)$ & $(15.2030)$ & (11.0107) & $(12.3119)$ & $(18.6781)$ \\
\hline & $0.5^{5}$ & $0.5^{5}$ & 0.9594 & 0.9791 & 0.9807 & 0.9848 & 0.9862 & 0.9987 \\
\hline & & & $(0.7720)$ & $(0.8589)$ & $(0.8767)$ & $(0.8540)$ & $(0.8717)$ & $(1.2557)$ \\
\hline & & $1.0^{5}$ & 0.9546 & 0.9635 & 0.9664 & 0.9793 & 0.9816 & 0.9947 \\
\hline & & & (1.7331) & (1.7739) & $(1.8229)$ & $(1.7555)$ & $(1.8035)$ & $(2.4495)$ \\
\hline & & $2.0^{5}$ & 0.9527 & 0.9540 & 0.9582 & 0.9825 & 0.9852 & 0.9848 \\
\hline & & & $(6.0963)$ & $(6.0429)$ & $(6.2535)$ & $(5.9008)$ & (6.1028) & (7.6733) \\
\hline & & $0.5^{2}: 1.0: 2.0^{2}$ & 0.9527 & 0.9579 & 0.9613 & 0.9645 & 0.9681 & 0.9884 \\
\hline & & & (3.1078) & $(3.4067)$ & $(3.5167)$ & (3.1961) & (3.2916) & (4.3313) \\
\hline
\end{tabular}


Table 3. (Continued).

\begin{tabular}{|c|c|c|c|c|c|c|c|c|}
\hline \multirow{2}{*}{$n_{1}: \ldots: n_{5}$} & \multirow{2}{*}{$\delta_{1(1)}: \ldots: \delta_{5(1)}$} & \multirow{2}{*}{$\sigma_{1}^{2}: \ldots: \sigma_{5}^{2}$} & \multicolumn{6}{|c|}{ Coverage probabilities (Expected lengths) } \\
\hline & & & FGCI & B.Jrule-E & B.Uni-E & B.Jrule-C & B.Uni-C & MOVER \\
\hline \multirow[t]{8}{*}{$100^{5}$} & \multirow[t]{8}{*}{$0.8^{5}$} & \multirow[t]{2}{*}{$0.5^{5}$} & 0.9542 & 0.9670 & 0.9691 & 0.9721 & 0.9742 & 0.9966 \\
\hline & & & $(0.5010)$ & $(0.5295)$ & $(0.5374)$ & $(0.5269)$ & $(0.5348)$ & $(0.7392)$ \\
\hline & & \multirow[t]{2}{*}{$1.0^{5}$} & 0.9509 & 0.9544 & 0.9570 & 0.9665 & 0.9688 & 0.9875 \\
\hline & & & $(1.0812)$ & $(1.0918)$ & $(1.1102)$ & $(1.0842)$ & (1.1024) & $(1.3890)$ \\
\hline & & \multirow[t]{2}{*}{$2.0^{5}$} & 0.9506 & 0.9507 & 0.9533 & 0.9730 & 0.9752 & 0.9755 \\
\hline & & & $(3.4721)$ & $(3.4569)$ & $(3.5238)$ & (3.4111) & $(3.4762)$ & $(4.0692)$ \\
\hline & & \multirow[t]{2}{*}{$0.5^{2}: 1.0: 2.0^{2}$} & 0.9500 & 0.9524 & 0.9552 & 0.9593 & 0.9619 & 0.9806 \\
\hline & & & (1.8380) & $(2.0041)$ & $(2.0416)$ & $(1.9233)$ & (1.9575) & $(2.3760)$ \\
\hline \multirow[t]{16}{*}{$25^{2}: 50: 100^{2}$} & \multirow[t]{8}{*}{$0.5^{5}$} & \multirow[t]{2}{*}{$0.5^{5}$} & 0.9608 & 0.9770 & 0.9830 & 0.9830 & 0.9886 & 0.9985 \\
\hline & & & $(1.5163)$ & $(1.4923)$ & $(1.6614)$ & $(1.4314)$ & $(1.5704)$ & $(2.2909)$ \\
\hline & & \multirow{2}{*}{$1.0^{5}$} & 0.9549 & 0.9595 & 0.9688 & 0.9785 & 0.9852 & 0.9940 \\
\hline & & & $(4.4104)$ & (3.8914) & $(4.6543)$ & (3.4894) & $(4.0239)$ & $(5.7364)$ \\
\hline & & \multirow[t]{2}{*}{$2.0^{5}$} & 0.9511 & 0.9484 & 0.9600 & 0.9813 & 0.9872 & 0.9826 \\
\hline & & & $(32.7160)$ & $(25.3223)$ & $(37.4111)$ & (17.8405) & $(23.1773)$ & (37.9900) \\
\hline & & \multirow[t]{2}{*}{$0.5^{2}: 1.0: 2.0^{2}$} & 0.9552 & 0.9603 & 0.9655 & 0.9801 & 0.9835 & 0.9901 \\
\hline & & & $(3.8324)$ & $(4.0083)$ & $(4.2595)$ & $(3.8460)$ & $(4.0906)$ & $(5.3939)$ \\
\hline & \multirow[t]{8}{*}{$0.8^{5}$} & \multirow[t]{2}{*}{$0.5^{5}$} & 0.9555 & 0.9656 & 0.9719 & 0.9753 & 0.9817 & 0.9959 \\
\hline & & & $(0.9159)$ & $(0.8965)$ & $(0.9522)$ & $(0.8736)$ & $(0.9239)$ & $(1.2760)$ \\
\hline & & \multirow{2}{*}{$1.0^{5}$} & 0.9506 & 0.9512 & 0.9583 & 0.9707 & 0.9774 & 0.9856 \\
\hline & & & $(2.2438)$ & (2.0817) & $(2.2545)$ & $(\mathbf{1 . 9 6 8 2})$ & $(2.1113)$ & $(2.7304)$ \\
\hline & & \multirow[t]{2}{*}{$2.0^{5}$} & 0.9496 & 0.9469 & 0.9551 & 0.9774 & 0.9824 & 0.9744 \\
\hline & & & $(10.1646)$ & $(8.9783)$ & $(10.2014)$ & $(7.7693)$ & $(8.6055)$ & (11.2231) \\
\hline & & \multirow[t]{2}{*}{$0.5^{2}: 1.0: 2.0^{2}$} & 0.9515 & 0.9536 & 0.9582 & 0.9704 & 0.9737 & 0.9832 \\
\hline & & & $(2.2063)$ & $(2.3189)$ & $(2.3964)$ & $(2.2595)$ & $(2.3367)$ & (2.8768) \\
\hline
\end{tabular}

Note: $25^{5}$ represents 25:25:25:25:25.

Table 4. The coverage probabilities and expected lengths for the $95 \%$ SCIs and credible intervals for all pairwise differences between the CVs of delta-lognormal distributions for $k=10$.

\begin{tabular}{|c|c|c|c|c|c|c|c|c|}
\hline \multirow{2}{*}{$n_{1}: \ldots: n_{10}$} & \multirow{2}{*}{$\delta_{1(1)}: \ldots: \delta_{10(1)}$} & \multirow{2}{*}{$\sigma_{1}^{2}: \ldots: \sigma_{10}^{2}$} & \multicolumn{6}{|c|}{ Coverage probabilities (Expected lengths) } \\
\hline & & & FGCI & B.Jrule-E & B.Uni-E & B.Jrule-C & B.Uni-C & MOVER \\
\hline \multirow[t]{6}{*}{$25^{10}$} & $0.5^{10}$ & $0.5^{10}$ & $\begin{array}{c}\mathbf{0 . 9 6 4 4} \\
(2.1718)\end{array}$ & $\begin{array}{c}\mathbf{0 . 9 7 9 6} \\
(2.1549)\end{array}$ & $\begin{array}{c}\mathbf{0 . 9 8 5 2} \\
(2.5017)\end{array}$ & $\begin{array}{c}0.9958 \\
(2.0864)\end{array}$ & $\begin{array}{c}\mathbf{0 . 9 9 8 1} \\
(2.4056)\end{array}$ & $\begin{array}{c}\mathbf{0 . 9 9 8 7} \\
(3.4437)\end{array}$ \\
\hline & & $1.0^{10}$ & $\begin{array}{c}\mathbf{0 . 9 5 5 9} \\
(6.9095)\end{array}$ & $\begin{array}{c}\mathbf{0 . 9 5 9 6} \\
(6.2680)\end{array}$ & $\begin{array}{c}\mathbf{0 . 9 7 1 7} \\
(7.9343)\end{array}$ & $\begin{array}{c}0.9952 \\
(5.7342)\end{array}$ & $\begin{array}{c}\mathbf{0 . 9 9 8 2} \\
(7.1201)\end{array}$ & $\begin{array}{c}\mathbf{0 . 9 9 3 5} \\
(9.6381)\end{array}$ \\
\hline & & $2.0^{10}$ & $\begin{array}{c}\mathbf{0 . 9 5 1 3} \\
(64.2109)\end{array}$ & $\begin{array}{c}0.9466 \\
(51.6243)\end{array}$ & $\begin{array}{c}\mathbf{0 . 9 6 2 7} \\
(83.5403)\end{array}$ & $\begin{array}{c}\mathbf{0 . 9 9 7 0} \\
(37.5819)\end{array}$ & $\begin{array}{c}\mathbf{0 . 9 9 9 0} \\
(54.7163)\end{array}$ & $\begin{array}{c}\mathbf{0 . 9 8 3 2} \\
(81.8119)\end{array}$ \\
\hline & $0.8^{10}$ & $0.5^{10}$ & $\begin{array}{c}\mathbf{0 . 9 5 5 8} \\
(1.2509)\end{array}$ & $\begin{array}{c}\mathbf{0 . 9 6 4 5} \\
(1.2566)\end{array}$ & $\begin{array}{c}\mathbf{0 . 9 7 3 3} \\
(1.3621)\end{array}$ & $\begin{array}{c}0.9836 \\
(1.2369)\end{array}$ & $\begin{array}{c}\mathbf{0 . 9 8 9 9} \\
(1.3398) \\
\end{array}$ & $\begin{array}{c}\mathbf{0 . 9 9 5 8} \\
(1.8373) \\
\end{array}$ \\
\hline & & $1.0^{10}$ & $\begin{array}{c}\mathbf{0 . 9 5 1 8} \\
(3.2511)\end{array}$ & $\begin{array}{c}\mathbf{0 . 9 5 1 3} \\
(3.1246)\end{array}$ & $\begin{array}{c}\mathbf{0 . 9 6 2 1} \\
(3.4760)\end{array}$ & $\begin{array}{c}0.9855 \\
(3.0068)\end{array}$ & $\begin{array}{c}\mathbf{0 . 9 9 1 4} \\
(3.3358)\end{array}$ & $\begin{array}{c}\mathbf{0 . 9 8 7 1} \\
(4.2290)\end{array}$ \\
\hline & & $2.0^{10}$ & $\begin{array}{c}\mathbf{0 . 9 5 1 1} \\
(16.2516)\end{array}$ & $\begin{array}{c}0.9468 \\
(15.1258)\end{array}$ & $\begin{array}{c}\mathbf{0 . 9 5 8 1} \\
(17.7445)\end{array}$ & $\begin{array}{c}0.9931 \\
(13.5223)\end{array}$ & $\begin{array}{c}\mathbf{0 . 9 9 6 2} \\
(15.6914)\end{array}$ & $\begin{array}{c}\mathbf{0 . 9 7 5 9} \\
(19.6085)\end{array}$ \\
\hline \multirow[t]{9}{*}{$50^{10}$} & $0.2^{10}$ & $0.5^{10}$ & $\begin{array}{c}\mathbf{0 . 9 6 7 8} \\
(4.3466)\end{array}$ & $\begin{array}{c}\mathbf{0 . 9 8 5 9} \\
(4.1581)\end{array}$ & $\begin{array}{c}\mathbf{0 . 9 8 9 2} \\
(5.2771)\end{array}$ & $\begin{array}{c}0.9988 \\
(3.9368)\end{array}$ & $\begin{array}{c}\mathbf{0 . 9 9 9 5} \\
(4.8806)\end{array}$ & $\begin{array}{c}\mathbf{0 . 9 9 9 3} \\
(7.2207)\end{array}$ \\
\hline & & $1.0^{10}$ & $\begin{array}{c}\mathbf{0 . 9 6 0 0} \\
(17.6759)\end{array}$ & $\begin{array}{c}\mathbf{0 . 9 6 8 0} \\
(14.5839)\end{array}$ & $\begin{array}{c}\mathbf{0 . 9 7 8 4} \\
(22.8034)\end{array}$ & $\begin{array}{c}0.9988 \\
(12.3672)\end{array}$ & $\begin{array}{c}\mathbf{0 . 9 9 9 7} \\
(17.8805)\end{array}$ & $\begin{array}{c}\mathbf{0 . 9 9 6 6} \\
(25.7964)\end{array}$ \\
\hline & & $2.0^{10}$ & $\begin{array}{c}\mathbf{0 . 9 5 2 5} \\
(584.809)\end{array}$ & $\begin{array}{c}0.9491 \\
(314.782)\end{array}$ & $\begin{array}{c}\mathbf{0 . 9 6 7 1} \\
(1958.586)\end{array}$ & $\begin{array}{c}0.9988 \\
(130.579)\end{array}$ & $\begin{array}{c}\mathbf{0 . 9 9 9 7} \\
(314.105)\end{array}$ & $\begin{array}{c}\mathbf{0 . 9 8 8 1} \\
(825.014)\end{array}$ \\
\hline & $0.5^{10}$ & $0.5^{10}$ & $\begin{array}{c}0.9614 \\
(1.2078)\end{array}$ & $\begin{array}{c}\mathbf{0 . 9 8 0 0} \\
(1.2981)\end{array}$ & $\begin{array}{c}\mathbf{0 . 9 8 2 8} \\
(1.3643)\end{array}$ & $\begin{array}{c}\mathbf{0 . 9 9 0 5} \\
(1.2842)\end{array}$ & $\begin{array}{c}\mathbf{0 . 9 9 2 7} \\
(1.3493)\end{array}$ & $\begin{array}{c}\mathbf{0 . 9 9 8 9} \\
(1.9553)\end{array}$ \\
\hline & & $1.0^{10}$ & $\begin{array}{c}\mathbf{0 . 9 5 4 3} \\
(2.9985)\end{array}$ & $\begin{array}{c}\mathbf{0 . 9 6 1 4} \\
(2.9750)\end{array}$ & $\begin{array}{c}\mathbf{0 . 9 6 7 6} \\
(3.1898)\end{array}$ & $\begin{array}{c}0.9874 \\
(2.8975)\end{array}$ & $\begin{array}{c}\mathbf{0 . 9 9 0 9} \\
(3.1028)\end{array}$ & $\begin{array}{c}\mathbf{0 . 9 9 4 2} \\
(4.2323)\end{array}$ \\
\hline & & $2.0^{10}$ & $\begin{array}{c}\mathbf{0 . 9 5 0 5} \\
(13.0586)\end{array}$ & $\begin{array}{c}0.9497 \\
(12.5061)\end{array}$ & $\begin{array}{c}\mathbf{0 . 9 5 7 7} \\
(13.7967)\end{array}$ & $\begin{array}{c}0.9915 \\
(11.6493)\end{array}$ & $\begin{array}{c}\mathbf{0 . 9 9 4 4} \\
(12.7980)\end{array}$ & $\begin{array}{c}\mathbf{0 . 9 8 3 4} \\
(16.6309)\end{array}$ \\
\hline & $0.8^{10}$ & $0.5^{10}$ & $\begin{array}{c}0.9543 \\
(0.7572)\end{array}$ & $\begin{array}{c}\mathbf{0 . 9 6 5 3} \\
(0.7873)\end{array}$ & $\begin{array}{c}\mathbf{0 . 9 6 9 9} \\
(0.8131)\end{array}$ & $\begin{array}{c}\mathbf{0 . 9 7 6 1} \\
(0.7817)\end{array}$ & $\begin{array}{c}\mathbf{0 . 9 7 9 9} \\
(0.8075)\end{array}$ & $\begin{array}{c}\mathbf{0 . 9 9 6 0} \\
(1.1153)\end{array}$ \\
\hline & & $1.0^{10}$ & $\begin{array}{c}\mathbf{0 . 9 5 1 0} \\
(1.7261)\end{array}$ & $\begin{array}{c}\mathbf{0 . 9 5 3 0} \\
(1.7192)\end{array}$ & $\begin{array}{c}\mathbf{0 . 9 5 8 4} \\
(1.7869)\end{array}$ & $\begin{array}{c}0.9747 \\
(1.6958)\end{array}$ & $\begin{array}{c}\mathbf{0 . 9 7 9 1} \\
(1.7624)\end{array}$ & $\begin{array}{c}\mathbf{0 . 9 8 7 2} \\
(2.2287)\end{array}$ \\
\hline & & $2.0^{10}$ & $\begin{array}{c}\mathbf{0 . 9 5 0 0} \\
(6.2757) \\
\end{array}$ & $\begin{array}{c}0.9485 \\
(6.1553) \\
\end{array}$ & $\begin{array}{c}\mathbf{0 . 9 5 4 1} \\
(6.4591) \\
\end{array}$ & $\begin{array}{c}.9835 \\
(5.9506) \\
\end{array}$ & $\begin{array}{c}\mathbf{0 . 9 8 6 6} \\
(6.2399) \\
\end{array}$ & $\begin{array}{c}\mathbf{0 . 9 7 4 9} \\
(7.4567) \\
\end{array}$ \\
\hline \multirow[t]{3}{*}{$100^{10}$} & $0.2^{10}$ & $0.5^{10}$ & $\begin{array}{c}0.9658 \\
(2.1038)\end{array}$ & $\begin{array}{c}\mathbf{0 . 9 8 6 8} \\
(2.2979)\end{array}$ & $\begin{array}{c}\mathbf{0 . 9 8 8 5} \\
(2.4450)\end{array}$ & $\begin{array}{c}\mathbf{0 . 9 9 5 7} \\
(2.2660)\end{array}$ & $\begin{array}{c}\mathbf{0 . 9 9 6 8} \\
(2.4078)\end{array}$ & $\begin{array}{c}\mathbf{0 . 9 9 9 4} \\
(3.5707)\end{array}$ \\
\hline & & $1.0^{10}$ & $\begin{array}{c}\mathbf{0 . 9 5 7 3} \\
(5.5609)\end{array}$ & $\begin{array}{c}\mathbf{0 . 9 6 8 4} \\
(5.4793)\end{array}$ & $\begin{array}{c}\mathbf{0 . 9 7 3 8} \\
(6.0256)\end{array}$ & $\begin{array}{c}\mathbf{0 . 9 9 3 7} \\
(5.2730)\end{array}$ & $\begin{array}{c}\mathbf{0 . 9 9 5 9} \\
(5.7794)\end{array}$ & $\begin{array}{c}\mathbf{0 . 9 9 6 8} \\
(8.2780)\end{array}$ \\
\hline & & $2.0^{10}$ & $\begin{array}{c}\mathbf{0 . 9 5 1 5} \\
(27.8994)\end{array}$ & $\begin{array}{c}\mathbf{0 . 9 5 2 3} \\
(26.0553)\end{array}$ & $\begin{array}{c}\mathbf{0 . 9 6 1 2} \\
(30.1289)\end{array}$ & $\begin{array}{c}0.9950 \\
(23.3884)\end{array}$ & $\begin{array}{c}\mathbf{0 . 9 9 7 2} \\
(26.7565)\end{array}$ & $\begin{array}{c}\mathbf{0 . 9 8 8 1} \\
(37.1355)\end{array}$ \\
\hline
\end{tabular}


Table 4. (Continued).

\begin{tabular}{|c|c|c|c|c|c|c|c|c|}
\hline \multirow{2}{*}{$n_{1}: \ldots: n_{10}$} & \multirow{2}{*}{$\delta_{1(1)}: \ldots: \delta_{10(1)}$} & \multirow{2}{*}{$\sigma_{1}^{2}: \ldots: \sigma_{10}^{2}$} & \multicolumn{6}{|c|}{ Coverage probabilities (Expected lengths) } \\
\hline & & & FGCI & B.Jrule-E & B.Uni-E & B.Jrule-C & B.Uni-C & MOVER \\
\hline \multirow[t]{12}{*}{$100^{10}$} & \multirow[t]{6}{*}{$0.5^{10}$} & \multirow[t]{2}{*}{$0.5^{10}$} & 0.9595 & 0.9799 & 0.9813 & 0.9856 & 0.9869 & 0.9989 \\
\hline & & & $(0.7720)$ & $(0.8589)$ & $(0.8767)$ & $(0.8540)$ & $(0.8718)$ & (1.2559) \\
\hline & & \multirow[t]{2}{*}{$1.0^{10}$} & 0.9536 & 0.9622 & 0.9653 & 0.9784 & 0.9810 & 0.9945 \\
\hline & & & (1.7364) & (1.7768) & (1.8256) & (1.7582) & (1.8066) & $(2.4531)$ \\
\hline & & \multirow[t]{2}{*}{$2.0^{10}$} & 0.9505 & 0.9518 & 0.9557 & 0.9815 & 0.9841 & 0.9838 \\
\hline & & & (6.1121) & $(6.0620)$ & $(6.2702)$ & (5.9173) & (6.1188) & (7.6931) \\
\hline & \multirow[t]{6}{*}{$0.8^{10}$} & \multirow[t]{2}{*}{$0.5^{10}$} & 0.9541 & 0.9668 & 0.9689 & 0.9720 & 0.9741 & 0.9964 \\
\hline & & & $(0.5013)$ & $(0.5298)$ & $(0.5376)$ & $(0.5272)$ & $(0.5351)$ & $(0.7395)$ \\
\hline & & \multirow[t]{2}{*}{$1.0^{10}$} & 0.9508 & 0.9542 & 0.9569 & 0.9665 & 0.9689 & 0.9874 \\
\hline & & & (1.0804) & (1.0907) & (1.1089) & (1.0832) & (1.1014) & (1.3878) \\
\hline & & \multirow[t]{2}{*}{$2.0^{10}$} & 0.9503 & 0.9502 & 0.9530 & 0.9726 & 0.9747 & $\overline{0.9753}$ \\
\hline & & & $(3.4656)$ & $(3.4492)$ & $(3.5157)$ & (3.4036) & $(3.4692)$ & $(4.0610)$ \\
\hline
\end{tabular}

Note: $25^{10}$ represents 25:25:25:25:25:25:25:25:25:25

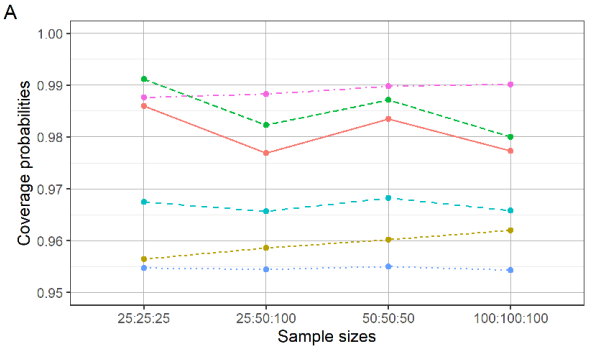

B
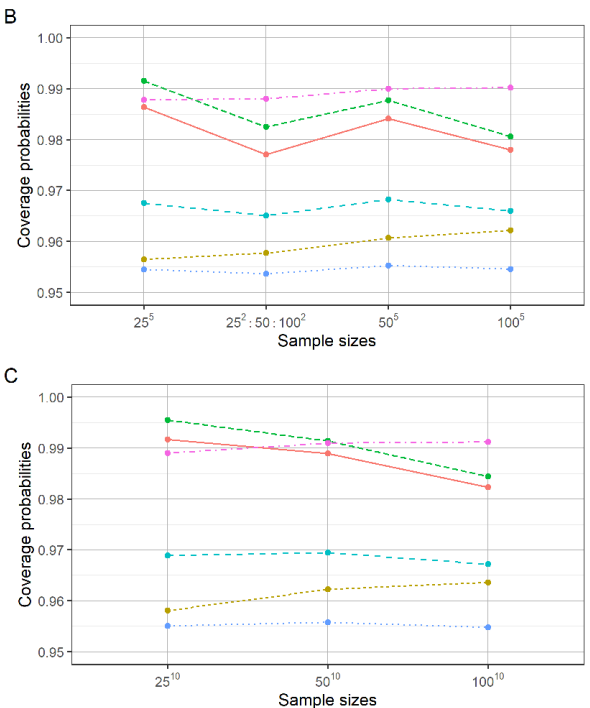

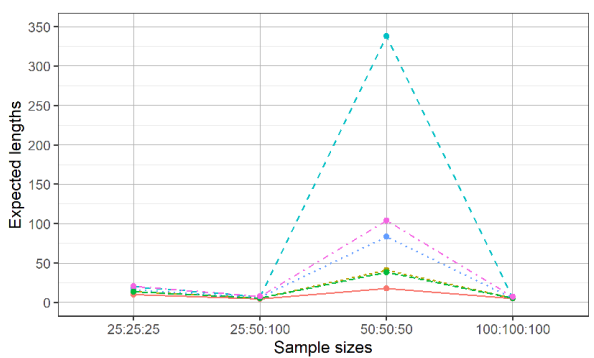

Methods

$\rightarrow$ B.Jrule-C

- B.Jrule-E

- B.Uni-C

- B.Uni-E

- FGCI

MOVER

Figure 1. Comparison of the performances of the proposed methods in terms of their coverage probabilities and expected lengths with various sample sizes: (A) $k=3$ (B) $k=5$ (C) $k=10$.

Table 5. AIC and BIC results for testing the distributions of the positive daily rainfall data from the five areas of Thailand in August 2020.

\begin{tabular}{|c|c|c|c|c|c|c|c|c|}
\hline \multirow{2}{*}{ Regions } & \multicolumn{4}{|c|}{$\overline{\mathrm{AIC}}$} & \multicolumn{4}{|c|}{$\overline{B I C}$} \\
\hline & Normal & Lognormal & Cauchy & Exponential & Normal & Lognormal & Cauchy & Exponential \\
\hline Northern & 200.1677 & 126.6431 & 154.5509 & 143.7143 & 202.3498 & 128.8252 & 156.7329 & 144.7143 \\
\hline Northeastern & 186.9685 & 145.5208 & 170.8114 & 151.8661 & 189.0576 & 147.6098 & 172.9005 & 152.9106 \\
\hline Central & 187.4002 & 142.8220 & 159.7491 & 148.7169 & 189.3916 & 144.8135 & 161.7405 & 149.7126 \\
\hline Eastern & 129.2900 & 99.5405 & 110.7899 & 100.7356 & 130.7061 & 100.9566 & 112.2060 & 101.4437 \\
\hline Southern & 140.0174 & 111.4550 & 124.1323 & 114.2896 & 141.4335 & 112.8711 & 125.5484 & 114.9977 \\
\hline
\end{tabular}


A

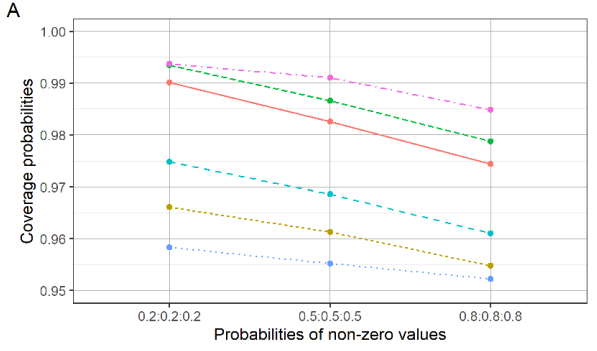

B
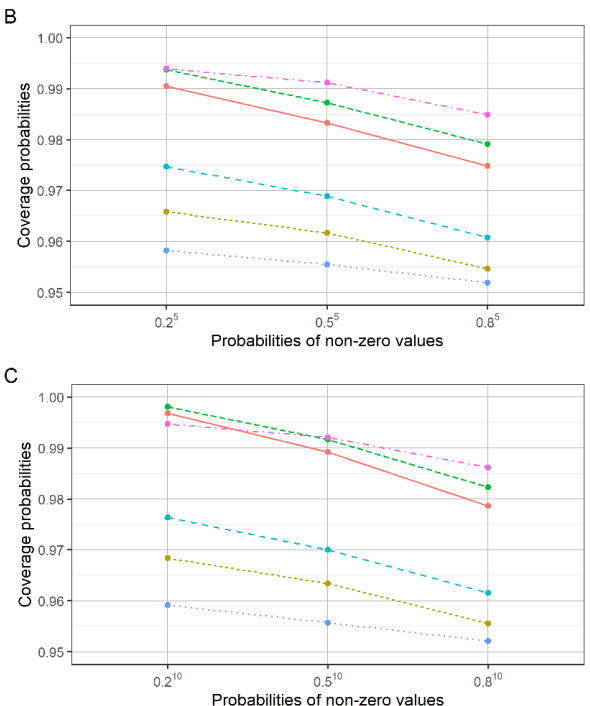

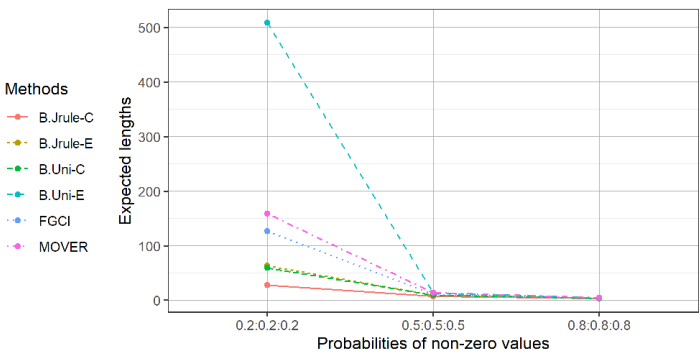

Methods

$\rightarrow$ B.Jrule-C

- B.Uni-C

- B. Uni-E

- MOVER

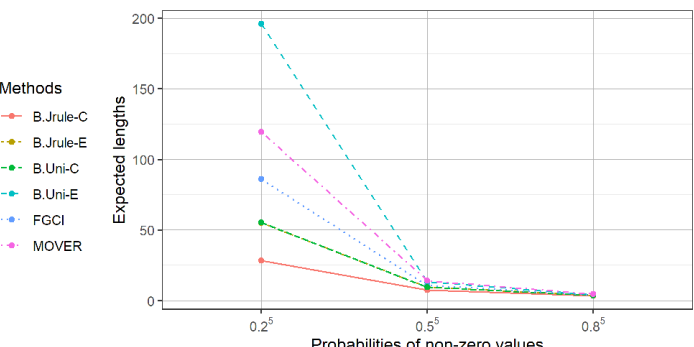

Probabilities of non-zero values

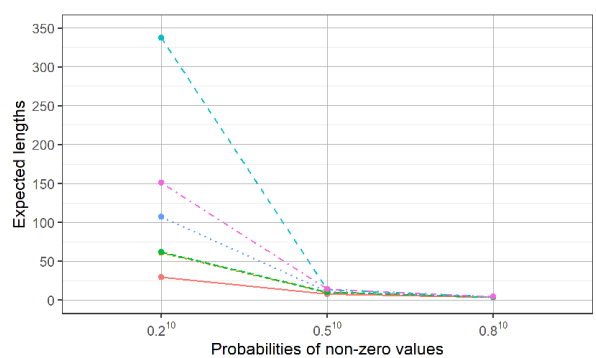

Methods

$\rightarrow$ B.Jrule-C

- B.Jrule-E

$\because$ B.Uni-C

- B.Uni-e

-. MOVER

Methods

$\rightarrow$ B.Jrule-C

- B.Jrule-E

- B. Uni-C

-\% B.Uni-E

MOVER

Figure 2. Comparison of the performances of the proposed methods in terms of their coverage probabilities and expected lengths with various probabilities of non-zero values: (A) $k=3(\mathrm{~B}) k=5$ (C) $k=10$.

Table 6. The $95 \%$ two-sided confidence intervals and credible intervals for all pairwise differences between the CVs of daily rainfall data from the five areas of Thailand in August 2020.

\begin{tabular}{lllllll}
\hline Regions & $C I_{F G C I}$ & $C I_{B . J r u l e-E}$ & $C I_{B . U n i-E}$ & $C I_{B . J r u l e-C}$ & $C I_{B . \text { Uni-C }}$ \\
\hline A1-A2 & {$[-18.6001,34.6174]$} & {$[-18.9230,29.7356]$} & {$[-17.4271,40.6785]$} & {$[-20.0641,27.7802]$} & {$[-22.5574,31.0130]$} & {$[-24.4099,41.5271]$} \\
A1-A3 & {$[-17.9489,34.8373]$} & {$[-14.7811,30.1761]$} & {$[-19.4179,40.0759]$} & {$[-18.8783,24.1259]$} & {$[-20.8181,36.4352]$} & {$[-24.6036,41.6372]$} \\
A1-A4 & {$[-12.0747,36.3137]$} & {$[-11.4058,31.0863]$} & {$[-13.2535,43.2976]$} & {$[-15.6054,24.9219]$} & {$[-18.9824,33.3564]$} & {$[-19.2874,42.6266]$} \\
A1-A5 & {$[-14.6835,36.5005]$} & {$[-12.4631,30.6305]$} & {$[-15.3529,42.0874]$} & {$[-15.9005,25.3713]$} & {$[-18.3991,35.6191]$} & {$[-20.8273,42.5263]$} \\
A2-A3 & {$[-19.1684,19.8007]$} & {$[-15.4068,20.8296]$} & {$[-21.9396,19.5370]$} & {$[-16.9424,18.6778]$} & {$[-18.6853,21.7661]$} & {$[-25.8643,25.7772]$} \\
A2-A4 & {$[-14.2284,20.9574]$} & {$[-12.1077,21.4176]$} & {$[-15.2508,20.4711]$} & {$[-12.3215,21.1200]$} & {$[-16.0818,19.4744]$} & {$[-20.5246,26.7455]$} \\
A2-A5 & {$[-15.1643,20.7719]$} & {$[-14.3124,21.0636]$} & {$[-16.9309,20.4638]$} & {$[-15.1223,19.4770]$} & {$[-15.7284,20.9116]$} & {$[-22.0733,26.6474]$} \\
A3-A4 & {$[-14.0533,20.3410]$} & {$[-12.8066,16.4067]$} & {$[-16.1177,22.6669]$} & {$[-13.4979,15.6852]$} & {$[-17.3305,21.2102]$} & {$[-20.6315,26.9413]$} \\
A3-A5 & {$[-15.2305,20.4287]$} & {$[-14.3490,16.8954]$} & {$[-17.6976,22.7643]$} & {$[-14.9963,16.0516]$} & {$[-16.0804,23.8267]$} & {$[-22.1806,26.8432]$} \\
A4-A5 & {$[-17.1536,14.8658]$} & {$[-15.0784,13.8954]$} & {$[-18.1792,16.4151]$} & {$[-14.9530,14.0968]$} & {$[-18.1763,16.4173]$} & {$[-23.1424,21.4920]$} \\
\hline
\end{tabular}




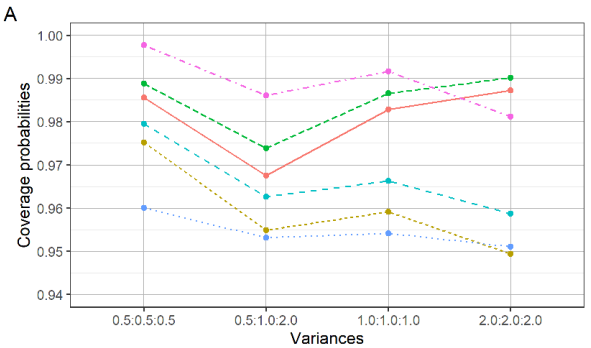

B
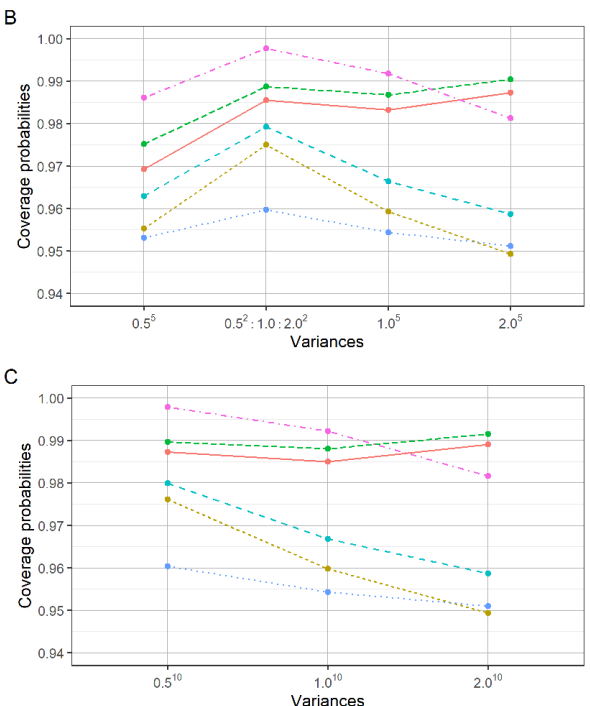
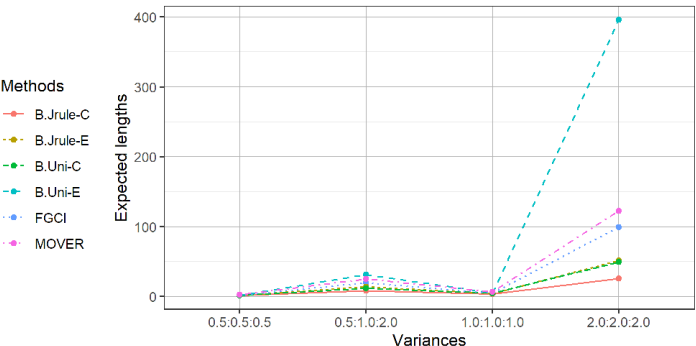

Methods

$\rightarrow$ B.Jrule-C

$\therefore$ B.Jrule-E

- B.Uni-C

- B.Uni-E

- FGCI

- mover

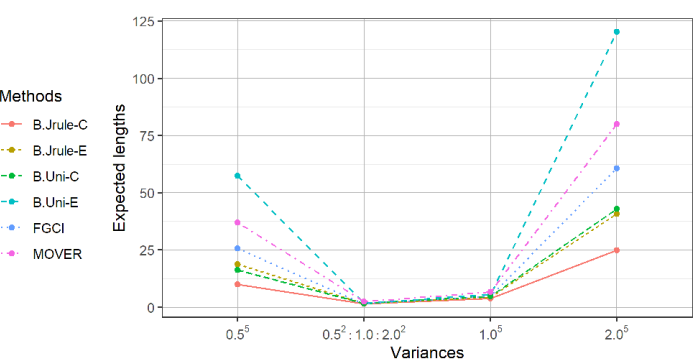

Methods

\#- B.Jrule-C

- B.Jrule-E

- B. Uni-C

- B.Unie

- MGCl

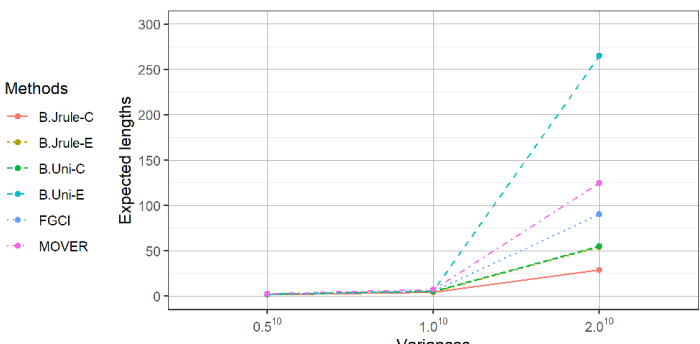

Methods

$\rightarrow$ B.Jrule-C

$\therefore$ B.Jrule-E

- B. Uni-C

B.Uni-e

- FGCI

Figure 3. Comparison of the performances of the proposed methods in terms of their coverage probabilities and expected lengths with various variances: (A) $k=3$ (B) $k=5$ (C) $k=10$.
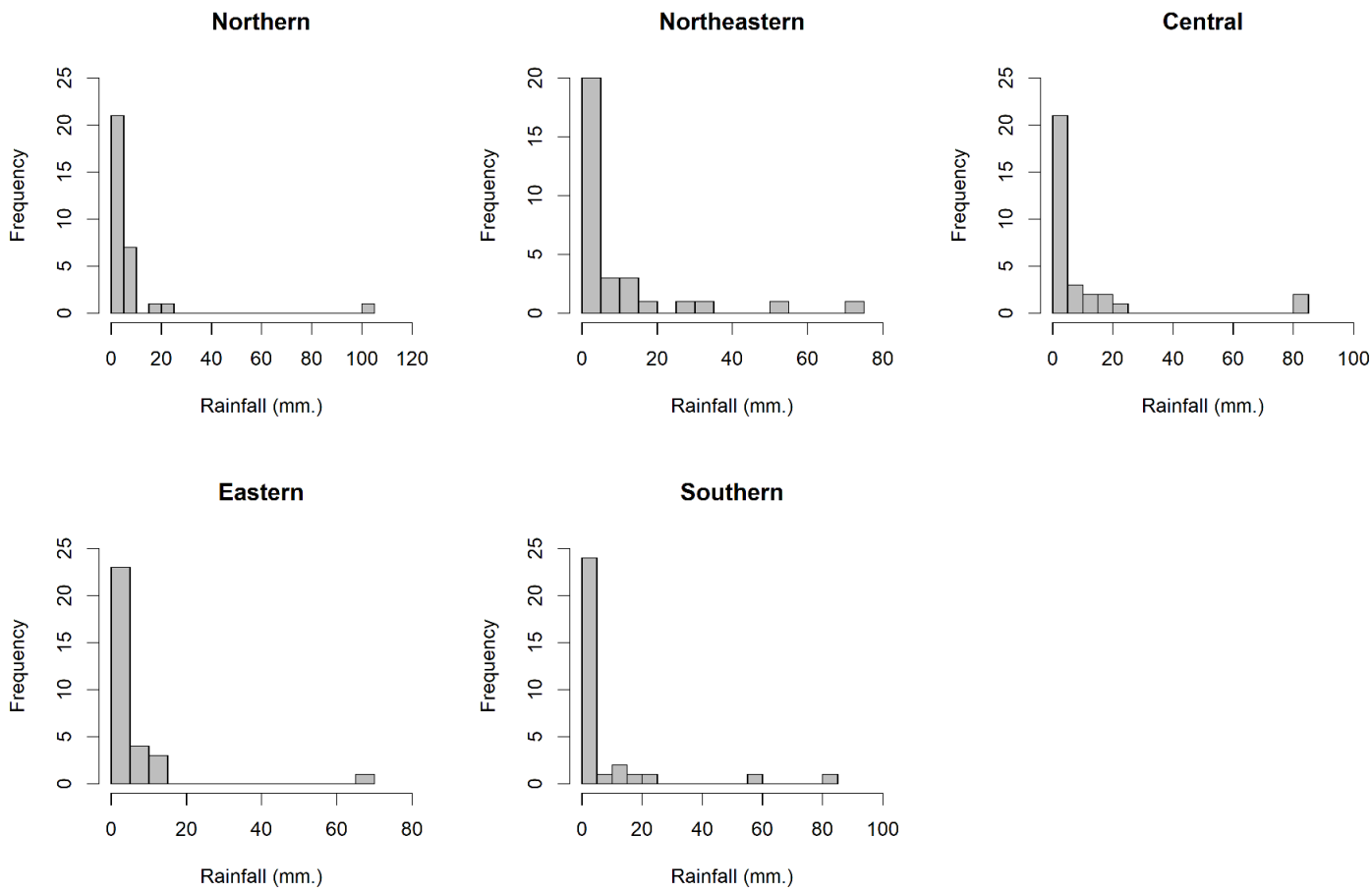

Figure 4. The density of daily rainfall data in the five areas of Thailand in August 2020. 
Northern

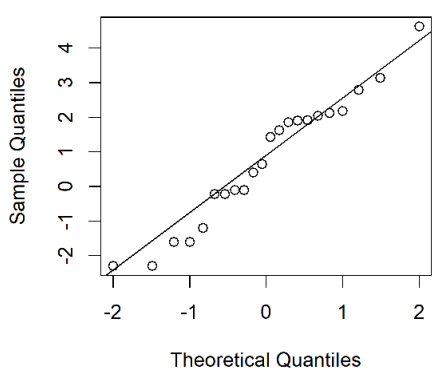

Eastern

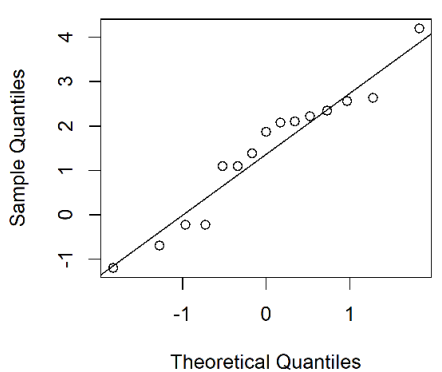

Northeastern

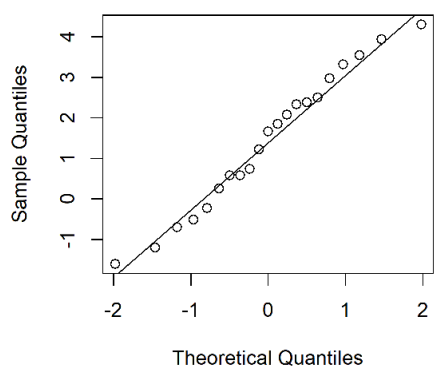

Southern

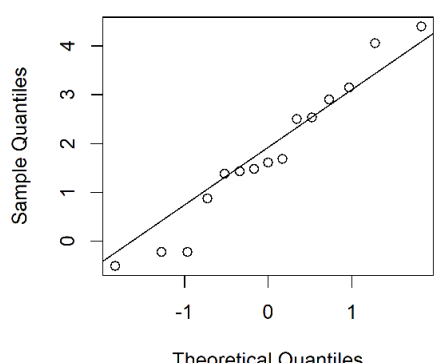

Central

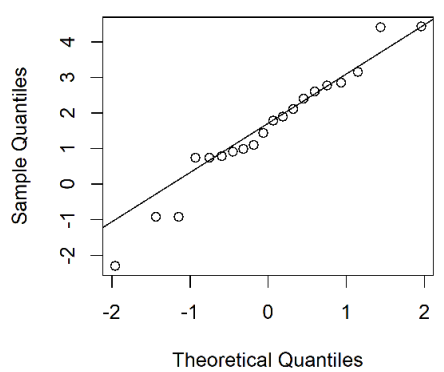

Theoretical Quantiles

Figure 5. Normal Q-Q plots of the log-transformed positive daily rainfall data from the five areas of Thailand in August 2020.

\section{DISCUSSION}

The simulation results indicate that the Bayesian credible interval using Jeffreys' rule prior outperformed the other methods in virtually all cases. Although the coverage probabilities in some cases were close to 1.00 , suggesting that overestimation may have occurred, the expected lengths were the shortest. Therefore, the Bayesian credible interval using Jeffreys' rule prior can be used to construct the SCIs for all of the pairwise differences between the CVs of delta-lognormal distributions. Since constructing SCIs concerns the differences between the parameters of interest for all pairwise comparisons, our findings correspond with Yosboonruang et al. (2020) who found that the highest posterior density Bayesian using Jeffreys' rule prior is appropriate for constructing the confidence interval for the difference between two independent CVs of delta-lognormal distributions. However, Abdel-Karim (2015) and Thangjai et al. (2019) reported that MOVER is the most suitable for constructing SCIs for the mean or CV of a lognormal distribution, but this is not in agreement with our findings for the data and scenario used in this study since the range of intervals for its SCI was wider than when using the Bayesian methods. In addition, the SCI range between the CVs of the daily rainfall data series from the five different areas of Thailand was too wide, and so this demonstrates that it is different in rainfall dispersion from five areas in Thailand.

\section{CONCLUSIONS}

Herein, we proposed methods to construct the SCIs for all pairwise differences between the CVs of delta-lognormal distributions, including FGCI, two Bayesian methods constructed under the equal-tailed confidence intervals and credible intervals using the Jeffreys' rule and uniform priors, and MOVER. The performances of the proposed methods were determined via their coverage probabilities together with their expected lengths under various circumstances. The results indicate that the Bayesian credible interval using the Jeffreys' rule prior was suitable for constructing the SCIs for all pairwise differences between the CVs of delta-lognormal distributions in terms of the coverage probability together with the expected length. Furthermore, FGCI is appropriate for constructing these SCIs in cases of the variances equal to 0.5 and 1.0 with the proportion of non-zero values equal to 0.5 and 0.8 for the sample sizes of 50 and 100 . In addition, the results of using daily rainfall data from five regions in Thailand coincided with those from 
the simulation study.

\section{REFERENCES}

Abdel-Karim, A. H. (2015). Construction of simultaneous confidence intervals for ratios of means of lognormal distributions. Communications in Statistics - Simulation and Computation, 44(2):271-283.

Aitchison, J. (1955). On the distribution of a positive random variable having a discrete probability mass at the origin. Journal of the American Statistical Association, 50:901-908.

Brown, L. D., Cai, T. T., and DasGupta, A. (2001). Interval estimation for a binomial proportion. Statistical Science, 16(2):101-117.

Buntao, N. and Niwitpong, S.-A. (2012). Confidence intervals for the difference of coefficients of variation for lognormal distributions and delta-lognormal distributions. Applied Mathematical Sciences, 6(134):6691-6704.

Buntao, N. and Niwitpong, S.-A. (2013). Confidence intervals for the ratio of coefficients of variation of delta-lognormal distribution. Applied Mathematical Sciences, 7(77):3811-3818.

Chen, Y.-H. and Zhou, X.-H. (2006). Generalized confidence intervals for the ratio or difference of two means for lognormal populations with zeros. UW Biostatistics Working Paper Series.

Donner, A. and Zou, G. Y. (2011). Estimating simultaneous confidence intervals for multiple contrasts of proportions by the method of variance estimates recovery. Statistics in Biopharmaceutical Research, 3(2):320-335.

Donner, A. and Zou, G. Y. (2012). Closed-form confidence intervals for functions of the normal mean and standard deviation. Statistical Methods in Medical Research, 21(4):347-359.

Fletcher, D. (2008). Confidence intervals for the mean of the delta-lognormal distribution. Environmental and Ecological Statistics, 15(2):175-189.

Fukuchi, H. (1988). Correlation properties of rainfall rates in the United Kingdom. Antennas and Propagation IEE Proceedings H - Microwaves, 135(2):83-88.

Hannig, J., Iyer, H., and Patterson, P. (2006). Fiducial generalized confidence intervals. Journal of the American Statistical Association, 101(473):254-269.

Hasan, M. S. and Krishnamoorthy, K. (2018). Confidence intervals for the mean and a percentile based on zero-inflated lognormal data. Journal of Statistical Computation and Simulation, 88(8):1499-1514.

Ingram Jr., G. W., Richards, W. J., Lamkin, J. T., and Muhling, B. (2010). Annual indices of Atlantic bluefin tuna (Thunnus thynnus) larvae in the Gulf of Mexico developed using delta-lognormal and multivariate models. Aquatic Living Resources, 23:35-47.

Jeffreys, H. (1946). An invariant form for the prior probability in estimation problems. Proceedings of the Royal Society of London. Series A. Mathematical and Physical Sciences, 186(1007):453-461.

Kedem, B. (1990). Estimation of mean rain rate: application to satellite observations. Journal of Geophysical Research, 95(D2):1965-1972.

Kong, C. Y., Jamaludin, S., Yusof, F., and Foo, H. M. (2012). Parameter estimation for bivariate mixed lognormal distribution. Journal of Science and Technology, 4(1):41-48.

Kvanli, A. H., Shen, Y. K., and Deng, L. Y. (1998). Construction of confidence intervals for the mean of a population containing many zero values. Journal of Business \& Economic Statistics, 16(3):362-368.

Li, J., Song, W., and Shi, J. (2015). Parametric bootstrap simultaneous confidence intervals for differences of means from several two-parameter exponential distributions. Statistics and Probability Letters, 106:39-45.

Li, X., Zhou, X., and Tian, L. (2013). Interval estimation for the mean of lognormal data with excess zeros. Statistics and Probability Letters, 83(11):2447-2453.

Mahmoudvand, R. and Hassani, H. (2009). Two new confidence intervals for the coefficient of variation in a normal distribution. Journal of Applied Statistics, 36(4):429-442.

Mandel, M. and Betensky, R. A. (2008). Simultaneous confidence intervals based on the percentile bootstrap approach. Computational statistics \& data analysis, 52(4):2158-2165.

Maneerat, P., Niwitpong, S.-A., and Niwitpong, S. (2018). Confidence intervals for the ratio of means of delta-lognormal distribution. In Anh, L. H., Dong, L. S., Kreinovich, V., and Thach, N. N., editors, Econometrics for Financial Applications, Studies in Computational Intelligence, pages 161-174. Springer International Publishing.

Maneerat, P., Niwitpong, S.-A., and Niwitpong, S. (2019a). Bayesian confidence intervals for a single 
mean and the difference between two means of delta-lognormal distributions. Communications in Statistics - Simulation and Computation, pages 1-29.

Maneerat, P., Niwitpong, S.-A., and Niwitpong, S. (2019b). Confidence intervals for the mean of deltalognormal distribution. In Kreinovich, V. and Sriboonchitta, S., editors, Structural Changes and their Econometric Modeling, Studies in Computational Intelligence, pages 264-274, Cham. Springer International Publishing.

Maneerat, P., Niwitpong, S.-A., and Niwitpong, S. (2020a). A bayesian approach to construct confidence intervals for comparing the rainfall dispersion in Thailand. PeerJ, 8:e8502.

Maneerat, P., Niwitpong, S.-A., and Niwitpong, S. (2020b). Bayesian confidence intervals for the difference between variances of delta-lognormal distributions. Biometrical Journal, pages 1-22.

Owen, W. J. and DeRouen, T. A. (1980). Estimation of the mean for lognormal data containing zeroes and left-censored values, with applications to the measurement of worker exposure to air contaminants. Biometrics, 36(4):707-719.

Sangnawakij, P. and Niwitpong, S.-A. (2017a). Confidence intervals for coefficients of variation in two-parameter exponential distributions. Communications in Statistics - Simulation and Computation, 46(8):6618-6630.

Sangnawakij, P. and Niwitpong, S.-A. (2017b). Confidence intervals for functions of coefficients of variation with bounded parameter spaces in two gamma distributions. Songklanakarin Journal of Science and Technology, 39(1):27-39.

Shimizu, K. (1993). A bivariate mixed lognormal distribution with an analysis of rainfall data. Journal of Applied Meteorology, 32(2):161-171.

Stone, J. V. (2013). Bayes' Rule: a tutorial introduction to Bayesian analysis. Sebtel Press, Sheffield.

Thai Meteorological Department (2015). The climate of Thailand. Available at https://www.tmd.go.th/en/archive/ thailand_climate.pdf. (accessed 30 August 2020).

Thangjai, W. and Niwitpong, S. (2020). Simultaneous confidence intervals for all differences of coefficients of variation of two-parameter exponential distributions. Thailand Statistician, 18(2):135-149.

Thangjai, W., Niwitpong, S.-A., and Niwitpong, S. (2019). Simultaneous confidence intervals for all differences of coefficients of variation of log-normal distributions. Hacettepe Journal of Mathematics \& Statistics, 48(5):1505-1521.

Tian, L. (2005). Inferences on the mean of zero-inflated lognormal data: the generalized variable approach. Statistics in Medicine, 24(20):3223-3232.

Tian, L. and Wu, J. (2006). Confidence intervals for the mean of lognormal data with excess zeros. Biometrical Journal. Biometrische Zeitschrift, 48(1):149-156.

Weerahandi, S. (1993). Generalized confidence interval. Journal of the American Statistical Association, 88(423):899-905.

Wilson, E. B. (1927). Probable inference, the law of succession, and statistical inference. Journal of the American Statistical Association, 22(158):209-212.

Wong, A. C. M. and Wu, J. (2002). Small sample asymptotic inference for the coefficient of variation: normal and nonnormal models. Journal of Statistical Planning and Inference, 104(1):73-82.

Wongkhao, A., Niwitpong, S.-A., and Niwitpong, S. (2015). Confidence intervals for the ratio of two independent coefficients of variation of normal distribution. Far East Journal of Mathematical Sciences, 98(6):741-757.

Wu, W.-H. and Hsieh, H.-N. (2014). Generalized confidence interval estimation for the mean of deltalognormal distribution: an application to New Zealand trawl survey data. Journal of Applied Statistics, 41(7):1471-1485.

Yosboonruang, N. and Niwitpong, S. (2020). Statistical inference on the ratio of delta-lognormal coefficients of variation. Applied Science and Engineering Progress, 8.

Yosboonruang, N., Niwitpong, S., and Niwitpong, S.-A. (2019a). Confidence intervals for coefficient of variation of three parameters delta-lognormal distribution. In Kreinovich, V. and Sriboonchitta, S., editors, Structural Changes and their Econometric Modeling, Studies in Computational Intelligence, pages 352-363. Springer International Publishing.

Yosboonruang, N., Niwitpong, S.-A., and Niwitpong, S. (2018). Confidence intervals for the coefficient of variation of the delta-lognormal distribution. In Anh, L. H., Dong, L. S., Kreinovich, V., and Thach, N. N., editors, Econometrics for Financial Applications, Studies in Computational Intelligence, pages 
327-337. Springer International Publishing.

Yosboonruang, N., Niwitpong, S.-A., and Niwitpong, S. (2019b). Measuring the dispersion of rainfall using Bayesian confidence intervals for coefficient of variation of delta-lognormal distribution: a study from Thailand. PeerJ, 7:e7344.

Yosboonruang, N., Niwitpong, S.-A., and Niwitpong, S. (2020). The Bayesian confidence intervals for measuring the difference between dispersions of rainfall in Thailand. PeerJ, 8:e9662.

Zhou, X. H. and Tu, W. (2000). Confidence intervals for the mean of diagnostic test charge data containing zeros. Biometrics, 56(4):1118-1125. 